\title{
Proof theory for functional modal logic
}

\begin{abstract}
We present some proof-theoretic results for the normal modal logic whose characteristic axiom is $\sim \square A \equiv \square \sim A$. We present a sequent system for this logic and a hypersequent system for its first-order form and show that these are equivalent to Hilbertstyle axiomatizations. We show that the question of validity for these logics reduces to that of classical tautologyhood and first-order logical truth, respectively. We close by proving equivalences with a Fitch-style proof system for revision theory.
\end{abstract}

Keywords: Functional modal logic, hypersequents, revision theory, proof theory

\section{Introduction and overview}

The modal logic obtained from adding the axiom scheme,

$$
\sim \square A \square \sim A,
$$

to the basic modal logic $\mathrm{K}$ is a simple modal logic. This logic is known as KD!, KDDc, or DAlt1. Its Kripke models have functional accessibility relations, that is, every world has a unique successor, or in symbols,

$$
\forall x \exists y(x R y \& \forall z(x R z \supset y=z)) .
$$

Segerberg (1986) presents an illuminating study of this logic, and others, using primarily model-theoretic means. The Hilbert-style axiom system for this logic is not amenable to proof-theoretic study. Wansing (1998, ch. 6) studies this logic in the context of display logic and relates it to propositional dynamic logic. Further alternative proof systems for it would be useful. The purpose of this paper is to provide sequent systems for this logic and to investigate some features of these systems.

The logic KD! and its extensions appears more frequently in philosophy than one would, at first, expect. It appears in the logic of imperatives of Parsons (2013). ${ }^{1}$ It appears in the logic of actuality of Davies and Humberstone

Presented by Heinrich Wansing; Received October 15, 2015

${ }^{1}$ Parsons uses an extension of the logic that adds the S4 axiom,

$$
\square A \supset \square \square A,
$$

to the logic. 
(1980). Actuality obeys the basic modal logical principles. The additional axiom scheme comes out by noting that if it is not the case that $A$ is actually true, then it is actually the case that $\sim A$ is true, and conversely. If we step away from classical logic briefly, we can find a functional accessibility relation, as in KD!, popping up in another place. Routley-Meyer models for relevant logics use the device known as the Routley star, *, to interpret negation. ${ }^{2}$ The Routley star is a function on worlds with the added condition that $w^{* *}=w$. The idea is that a world $w$ satisfies $\sim A$ just in case $w^{*}$ does not satisfy $A$. The Routley star gives a functional accessibility relation on worlds, as is required for $\mathrm{KD} !^{3}$ Locative logic, the modal logic of locations, is an extension of this logic. ${ }^{4}$ In locative logics, there are sets of operators, each indexed to a location, $\square_{n}$, which are read as 'holds at location $n$ '. These operators obey the axiom scheme given at the outset of the paper. Additionally, the logic has natural connections to the stratification used in the study of computational complexity in light linear logic. ${ }^{5}$

The logic shows up in another place, namely the revision theory of circular definitions. ${ }^{6}$ Revision theory uses sets $h$, called hypotheses, to interpret circularly defined predicates. The defining clause of the predicate is then used to revise the hypothesis to obtain a new hypothesis, $\Delta(h)$. Standefer (2015) extended basic revision theory with a unary operator, $\square$, that is intuitively understood as "prior to revision." The logic of this operator is $\mathrm{KD} !^{7}$ In fact, this operator obeys the quantified version of the modal logic with both directions of the Barcan formula. We will leave further details of revision theory to $\S 5$.

In this paper, we will explore the proof theory of the logic KD! and one of its quantified cousins. Although we have presented many places in which this modal logic shows up, the primary one, for our purposes, will be revision theory, which will show up again in $\S 5$. We will use the name $\mathrm{KD}$ ! for the propositional logic and QD! for the first-order logic with both directions of the Barcan formula. We will present a sequent system for KD! and sketch a proof of the cut elimination theorem for it (§2). The addition of

\footnotetext{
${ }^{2}$ See Restall (1999) and Dunn and Restall (2002), among others. for more on the Routley star.

${ }^{3}$ We would like to thank Dave Ripley for the preceding three examples.

${ }^{4}$ See Rescher and Urquhart (1971, ch. 2), there called 'topological logic', and Garson (2013, 52-54).

${ }^{5}$ See Baillot and Mazza (2010) and Boudes et al. (2015) for details on light linear logic. We thank Daniel Murfet for introducing me to this example.

${ }^{6}$ See Gupta and Belnap (1993) for a full exposition of revision theory.

${ }^{7}$ Standefer (2015) calls the logic RT.
} 
quantifiers reveals the system's shortcomings, motivating the development of a hypersequent system, which we investigate (§3). We prove equivalences between different formulations of the logics. We go on to show that KD! and QD! reduce, in a sense, to tautologies and first-order logical truths, respectively $(\S 4)$. Finally, we illustrate two connections between QD! and a proof system for revision theory $(\S 5)$. Let us begin with some background on modal logic.

\section{Background}

We will present some of the formal background for the modal logics in question. The sentences of the propositional logic will be defined as follows, where $A T$ is the set of atoms, $\{p, q, r \ldots\} .^{8}$

$$
A::=A T|\sim A|(A \& B)|(A \vee B)|(A \supset B) \mid \square A^{9}
$$

We will use a standard definition of a Kripke frame as a pair $(W, R)$, where $W \neq \emptyset$ and $R \subseteq W \times W$, and a Kripke model $M$ as a frame $(W, R)$ together with a true-in-a-world relation, $\Vdash \subseteq W \times L$, where $L$ is the set of sentences of the propositional language. ${ }^{10}$ The true-in-a-world relation, written as ' $w \Vdash A$ ' is defined as usual. ${ }^{11}$ In particular, the modal box has the following truth condition.

$$
w \Vdash \square A \text { iff } \forall w^{\prime}\left(w R w^{\prime} \Rightarrow w^{\prime} \Vdash A\right)
$$

Since the focus will be on frames with a functional accessibility relation, we can take $R$ to be a function, $W \mapsto W$, and rewrite the truth condition as follows.

$$
w \Vdash \square A \text { iff } R(w) \Vdash A
$$

The logic of frames with a functional accessibility relation is KD!, which is axiomatized by the axioms for $\mathrm{K}$, namely, all classical tautologies, the $\mathrm{K}$ axiom,

$$
\square(A \supset B) \supset(\square A \supset \square B)
$$

\footnotetext{
${ }^{8}$ The propositional constant, $\perp$, will not be included until $\S 5$. Until then, we will take $\perp$ to be defined as $p \& \sim p$, for some atom $p$. We also define $(A \equiv B)$ as usual as $(A \supset B) \&(B \supset A)$.

${ }^{9}$ To cut down on parentheses, we will adopt the convention that $\square$, $\sim$, and $\forall$, bind more tightly than \& and $\vee$, which in turn bind more tightly than $\supset$ and $\equiv$, dropping parentheses where possible.

${ }^{10}$ See, for example, Chellas (1980) or Blackburn et al. (2002) for more on Kripke models.

${ }^{11}$ We will sometimes write ' $M, w \Vdash A$ ' to emphasize the model $M$
} 
modus ponens, and the rule of Necessitation, from $\vdash A$ to infer $\vdash \square A$, together with the KD! axiom,

$$
\sim \square A \equiv \sim A .^{12}
$$

As can be shown by standard techniques, KD! is sound and complete with respect to Kripke frames with functional accessibility relations. ${ }^{13}$

Kripke models are generalized to the first-order case in a standard way. ${ }^{14}$ The first-order wffs will be defined as follows, where ATP is the set of atomic formulas, $\{F x, R y z, \ldots\}$, consisting of a predicate letter followed by a number of variables determined by the arity of the letter.

$$
A::=A T P|\sim A|(A \& B)|(A \vee B)|(A \supset B)|\forall x A| \square A
$$

The existential quantifier is taken as defined. Except when explicitly noted in $\S 5$, identity will not be in the language. In the first-order setting, a formula will be any wff and a sentence will be a formula with no free variables. ${ }^{15}$ The first-order logic QD! is obtained by adding to KD! quantifier axioms, the rule of generalization, and both the Barcan formula,

$$
\forall x \square A \supset \square \forall x A,
$$

and converse Barcan formula,

$$
\square \forall x A \supset \forall x \square A .{ }^{16}
$$

As is well known, models for both the Barcan and converse Barcan formulas have constant domains. In many applications, constant domain models are not desirable, since often one does not want to require that everything that

\footnotetext{
${ }^{12} \mathrm{~A}$ bimodal form of this axiom is $\square A \equiv \diamond A$, but for most of the paper, $\diamond$ will not be taken as primitive in the language.

${ }^{13}$ See, for example, Blackburn et al. (2002).

${ }^{14}$ See, for example, Hughes and Cresswell (1996, ch. 13).

${ }^{15}$ We will use 'sentence' for items in the propositional language as well.

${ }^{16}$ For concreteness, we add to KD! the following axiom schemes and the rule of generalization: from $\vdash A$ to infer $\vdash \forall x A$.

Q1 $\forall x(A \supset B) \supset(\forall x A \supset \forall x B)$

Q2 $\forall x A(x) \supset A(t)$, where $t$ is free for $x$

Q3 $A \supset \forall x A$, where $x$ is not free in $A$

We will also note that technically, only the Barcan formula needs to be added, as the converse is derivable, given our assumptions. We list both directions for emphasis.
} 
exists does so necessarily. For our purposes, constant domains are desirable because of the eventual connections we will draw between KD! and revision theory. The revision-theoretic box obeys the principle $\forall x \square A \equiv \square \forall x A$, as domains are constant from one revision to the next. Therefore, we will focus on models with constant domains, and so adopt both directions of the Barcan formula. ${ }^{17}$ We will also note that QD! is sound and complete with respect to constant domain first-order KD! frames, as can be shown with standard techniques. ${ }^{18}$

As a final bit of background on KD!, we list some of its theorems that will be useful throughout.

LEMMA 1. KD! has the following theorems.

1. $\square(A \& B) \equiv(\square A \& \square B)$

2. $\square(A \supset B) \equiv(\square A \supset \square B)$

3. $\square(A \vee B) \equiv(\square A \vee \square B)$

4. $\square A \vee \square \sim A$

5. $\sim \square \perp$

These can all be established by straightforward model-theoretic argument combined with the fact that $\mathrm{KD}$ ! is complete. As an illustration, take 3 . Suppose that $w \Vdash \square(A \vee B)$.

$$
\begin{array}{ccc}
w \Vdash \square(A \vee B) & \text { iff } & R(w) \Vdash A \vee B \\
& \text { iff } & R(w) \Vdash A \text { or } R(w) \Vdash B \\
\text { iff } & w \Vdash \square A \text { or } w \Vdash \square B \\
\text { iff } & w \Vdash \square A \vee \square B
\end{array}
$$

We shall end this section by mentioning a connection that may have occurred to the reader. The modal logic KD! coincides with the "neXt" fragment of linear temporal logic. ${ }^{19}$ This suggests further avenues that would be worth exploring. For example, linear temporal logic has been useful in the study of computation, and there are connections between revision and computation. ${ }^{20}$ We will, however, not pursue these ideas here.

\footnotetext{
${ }^{17}$ Investigation of first-order KD! minus one or both directions of the Barcan formula would be interesting. In this connection, the different quantifier rules of Fitting (2014) may be useful. Kawai (1987) provides methods for approaching the problem in the setting of infinitary logic. We will not further pursue the issue here. Thanks to an anonymous referee for pointing us towards these papers.

${ }^{18}$ See Hughes and Cresswell (1996).

${ }^{19}$ See Gabbay et al. (2003, §2.1) and Merz (1992), among others, for more on linear temporal logic. We would like to thank Kohei Kishida for suggesting this connection.

${ }^{20}$ For more on the latter connection, see Antonelli (1994).
} 
Shawn Standefer

\section{Sequent system}

Hilbert-style systems have their virtues, but they are not user-friendly. We will, then, provide a sequent system, LKD!, for KD! This system is obtained by adding one rule to a multiple conclusion sequent calculus for classical logic, which we outline here. We use ' $\Gamma$ ' and ' $\Delta$ ' to stand for multisets of sentences, i.e. sets of sentences that keep track of how many occurrences of each sentence they contain. We use ' $\Gamma, \Delta$ ' for the multiset union of $\Gamma$ and $\Delta$, and ' $\Gamma, A$ ' for the special case when $\Delta$ is the singleton of $A$. There is a single axiom,

$$
p \succ p
$$

as well as left and right rules for each connective. ${ }^{21}$

$$
\begin{array}{cc}
\frac{\Gamma \succ \Delta, A}{\sim A, \Gamma \succ \Delta}(\sim \succ) & \frac{A, B, \Gamma \succ \Delta}{A \& B, \Gamma \succ \Delta}(\& \succ) \\
\frac{A, \Gamma \succ \Delta}{\Gamma \succ \Delta, \sim A}(\succ \sim) & \frac{\Gamma \succ \Delta, A \quad \Sigma \succ \Theta, B}{\Gamma, \Sigma \succ \Delta, \Theta, A \& B}(\succ \&) \\
\frac{\Gamma \succ \Delta, A, B}{\Gamma \succ \Delta, A \vee B}(\succ \vee) & \frac{\Gamma, A \succ \Delta, B}{\Gamma \succ \Delta, A \supset B}(\succ \supset) \\
\frac{A, \Gamma \succ \Delta \quad B, \Sigma \succ \Theta}{A \vee B, \Gamma, \Sigma \succ \Delta, \Theta}(\vee \succ) & \frac{\Gamma \succ \Delta, A \quad B, \Sigma \succ \Theta}{A \supset B, \Gamma, \Sigma \succ \Delta, \Theta}(\supset \succ)
\end{array}
$$

It has the standard left and right structural rules, including weakening and contraction. ${ }^{22}$

$$
\begin{array}{ll}
\frac{\Gamma \succ \Delta}{A, \Gamma \succ \Delta}(K \succ) & \frac{\Gamma \succ \Delta}{\Gamma \succ \Delta, A}(\succ K) \\
\frac{A, A, \Gamma \succ \Delta}{A, \Gamma \succ \Delta}(W \succ) & \frac{\Gamma \succ \Delta, A, A}{\Gamma \succ \Delta, A}(\succ W)
\end{array}
$$

\footnotetext{
${ }^{21}$ These rules are multiplicative, or context-independent, rules. Given the structural rules of weakening and contraction, however, the additive, or context-sharing, rules are interderivable with these. See, for example, French and Ripley (2015) for details.

${ }^{22}$ The structural rules are labelled following the conventions of Restall (2000). Others, such as Troelstra and Schwichtenberg (2000), use $W$ and $C$, respectively, for the rules labelled here as $K$ and $W$.
} 
We will use the notation: $\square \Gamma$ for $\square A_{1}, \ldots, \square A_{n}$, where $\Gamma$ is $A_{1}, \ldots, A_{n}$. We add to this system the following rule to obtain LKD! ${ }^{23}$

$$
\frac{\Gamma \succ \Delta}{\square \Gamma \succ \square \Delta}(\square)
$$

We will note, without proof, that although the axiom rule is restricted to atoms, sequents of the form $A \succ A$ are derivable for all sentences $A$.

The modal rule $(\square)$ looks similar to the modal rule used by some proof systems for $\mathrm{K}$, but that rule requires that $\Delta$ contain exactly one sentence. ${ }^{24}$ Otherwise, the following is derivable.

$$
\frac{\frac{A \succ A}{\succ A, \sim A}(\succ \sim)}{\frac{\succ \square A, \square \sim A}{\succ \square A \vee \square \sim A}_{(\triangleright)}^{(\succ \vee)}}
$$

The final sequent is invalidated by the following $\mathrm{K}$ model. Let $W=\{w, u, v\}$ and suppose $w R u$ and $w R v$. Let $u \Vdash A$ and $v \Vdash \sim A$. Then $w \nVdash \square A \vee \square \sim A$. This sentence is, however, KD! valid. Using the ( $\square)$ rule, we can derive the commutation of the box with all the logical connectives. As an illustration, here is one of the derivations, which omits two weakening steps.

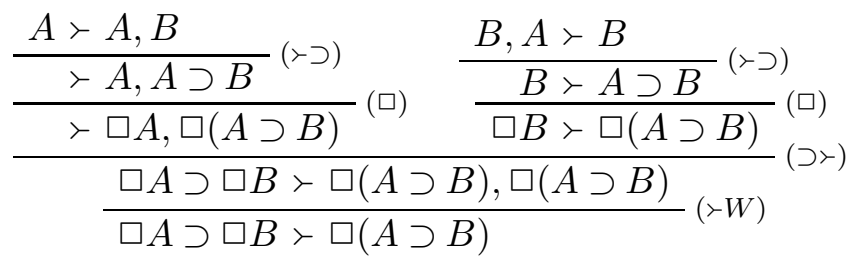

A rule is admissible in a sequent system whenever it is the case that if the premises are derivable, then the conclusion is also derivable. The cut elimination theorem says that the cut rule,

$$
\frac{\Gamma \succ \Delta, A \quad A, \Sigma \succ \Theta}{\Gamma, \Sigma \succ \Delta, \Theta}(c u t)
$$

is admissible. One sign that a sequent system is well-behaved is that it permits an elimination theorem. ${ }^{25}$ We can show that LKD! permits an elimination theorem. The proof proceeds via a standard approach of showing the admissibility of a stronger rule,

\footnotetext{
${ }^{23}$ This rule has also been proposed by Lahav and Zohar (2014). We would like to thank an anonymous referee for this reference.

${ }^{24}$ See, for example, Poggiolesi $(2010,45)$ and the citations there.

${ }^{25}$ For more on elimination theorems, see Gentzen (1964), Restall (2000), Troelstra and Schwichtenberg (2000), or Negri and von Plato (2001). The approach followed here is closest to that of Gentzen (1964).
} 


$$
\frac{\Gamma \succ \Delta, A \quad A, \Sigma \succ \Theta}{\Gamma, \Sigma^{-A} \succ \Delta^{-A}, \Theta}(\text { mix })
$$

where the notation $\Delta^{-A}$ stands for the multiset $\Delta$ with all occurrences of $A$ removed. The proof uses a small variation on Gentzen's technique of using a double induction on degree and rank of the mix. The degree of the mix is the number of logical connectives appearing in the mix formula, which is the displayed $A$ in the mix rule. The left rank of the mix is the length of the longest upwards path that starts from the left premiss of mix that does not cross an instance of the $(\square)$ rule and each sequent in the path contains the mix formula to the right of the turnstile. The right rank is defined similarly for the right premiss of mix. The rank of the mix is the sum of the left and right ranks of the mix. The rank is, then, calculated in the same way as for the classical sequent system, except that the $(\square)$ rule resets rank in its conclusion. For the proof sketch, we will need to define principal and parametric occurrences of sentences in proofs. An occurrence of a sentence is principal in an application of a rule $\rho$ just in case $\rho$ is an instance of a connective rule and the occurrence is the displayed one in the conclusion of the connective rule. An occurrence of a sentence is parametric in a rule just in case it is not principal.

Theorem 2.1 (Elimination Theorem). The sequent system LKD! has cut as an admissible rule.

Proof. The strategy of the proof is to permute instances of mix above rules, decreasing the left rank, until it is 1 , and then doing the same until the right rank is 1 . Then, mix is permuted above the rules introducing the mix formula into both premises. The proof is similar to proofs for the classical sequent calculus, so we will omit most of the cases. ${ }^{26}$ We will present one case here, the case in which the mix formula is $\square A$ and it was just introduced.

$$
\frac{\frac{\Gamma \succ \Delta, A}{\square \Gamma \succ \square \Delta, \square A} \text { (口) } \quad \frac{A, \Sigma \succ \Theta}{\square A, \square \Sigma \succ \square \Theta}}{(\square)} \text { (mix) }
$$

The mix can be carried out on simpler formulas as follows.

$$
\frac{\frac{\Gamma \succ \Delta, A \quad A, \Sigma \succ \Theta}{\Gamma, \Sigma^{-A} \succ \Delta^{-A}, \Theta} \text { (mix) }}{\square \Gamma, \square\left(\Sigma^{-A}\right) \succ \square\left(\Delta^{-A}\right), \square \Theta}(\square)
$$

\footnotetext{
${ }^{26}$ The omitted cases are handled in a way similar to that of Gentzen (1964). See Curry (1963) for a proof using the multiplicative rules, although the set up is slightly different than here.
} 
The multisets $\square\left(\Sigma^{-A}\right)$ and $(\square \Sigma)^{-\square A}$ are identical, as are $\square\left(\Delta^{-A}\right)$ and $(\square \Delta)^{-\square A}$, so the final lines of the two derivations match.

There is no case in which the $(\square)$ rule is followed by a cut on a sentence that was parametric in $(\square)$, as all sentences in the sequent are principal in that rule.

We conclude that in LKD! cut is an admissible rule.

Let $\mathrm{KD}$ ! $\vdash A$ mean that there is a KD! derivation of $A$. As one might expect, $\mathrm{LKD}$ ! is equivalent to $\mathrm{KD}$ ! in the following sense.

Theorem 2.2. 1. If $\mathrm{KD} ! \vdash A$ then the sequent $\succ A$ is derivable in $\mathrm{LKD}$ !

2. If $A_{1}, \ldots, A_{n} \succ B_{1}, \ldots, B_{m}$ is derivable in LKD!, then

$$
\mathrm{KD} ! \vdash\left(A_{1} \& \ldots \& A_{n}\right) \supset\left(B_{1} \vee \ldots \vee B_{m}\right)
$$

Proof. The proof of (1) is straightforward, given the Elimination Theorem. We will provide a derivation of the $\mathrm{K}$ axiom here.

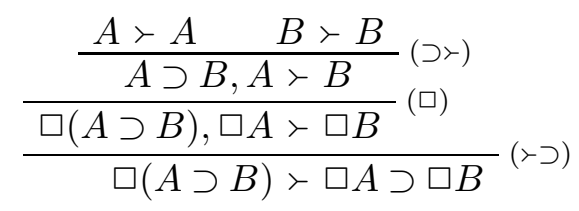

The proof of $(2)$ proceeds via an induction on the length of the proof. Since LKD! is based on a classical multiple conclusion sequent calculus, we will only present the modal rule step here. ${ }^{27}$

Suppose the last step of a proof is the following.

$$
\frac{A_{1}, \ldots, A_{n} \succ B_{1}, \ldots, B_{m}}{\square A_{1}, \ldots, \square A_{n} \succ \square B_{1}, \ldots, \square B_{m}}(\square)
$$

The KD! proof proceeds as follows.

$$
\begin{array}{ll}
A_{1} \& \ldots \& A_{n} \supset B_{1} \vee \ldots \vee B_{m} & \text { Inductive Hypothesis } \\
\square\left(A_{1} \& \ldots \& A_{n} \supset B_{1} \vee \ldots \vee B_{m}\right) & \text { Necessitation } \\
\square\left(A_{1} \& \ldots \& A_{n}\right) \supset \square\left(B_{1} \vee \ldots \vee B_{m}\right) & \mathrm{K} \text { axiom, modus ponens } \\
\square A_{1} \& \ldots \& \square A_{n} \supset \square\left(B_{1} \vee \ldots \vee B_{m}\right) & \text { lemma 1, transitivity } \\
\square A_{1} \& \ldots \& \square A_{n} \supset \square B_{1} \vee \ldots \vee \square B_{m} & \text { lemma 1, transitivity }
\end{array}
$$

The final line is the desired conclusion.

\footnotetext{
${ }^{27}$ See Bimbó (2014), for example, for details on similar cases for the classical connectives.
} 
In KD!, $\diamond$ is defined as $\sim \square \sim$. We can take $\diamond$ as a primitive along with $\square$ and use the following, alternative rule. ${ }^{28}$

$$
\frac{\Gamma, \Pi \succ \Delta, \Theta}{\square \Gamma, \diamond \Pi \succ \square \Delta, \diamond \Theta}(\square \diamond)
$$

Let $L K D !^{+}$be the system LKD! with $(\square \diamond)$ replacing $(\square)$. We have the following.

THeOREm 2.3. Cut is admissible for LKD! ${ }^{+}$.

$\mathrm{LKD}^{+}$yields the desired relationships between $\square$ and $\diamond$.

Lemma 2. The following are derivable in $\mathrm{LKD} !^{+}$.

1. $\square A \succ \sim \diamond \sim A$

2. $\sim \triangleright \sim A \succ \square A$

3. $\diamond A \succ \sim \square \sim A$

4. $\sim \square \sim A \succ \diamond A$

Proof. We will derive only the first, as derivations for the others are basically the same.

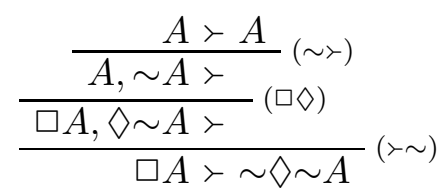

The equivalence between $\square$ and $\sim \diamond \sim$, and between $\diamond$ and $\sim \square \sim$, extends to cases in which these connectives are embedded, as well.

Finally, we connect LKD! and LKD! ${ }^{+}$.

THEOREM 2.4. Let $\Gamma \succ \Delta$ be a sequent, possibly containing $\diamond$, and let $\Gamma^{\prime} \succ \Delta^{\prime}$ be the sequent obtained by replacing all instances of $\diamond$ in $\Gamma$ and $\Delta$ with $\sim \square \sim$.

(1) If the sequent $\Gamma \succ \Delta$ is derivable in LKD!, $\Gamma \succ \Delta$ is derivable in LKD!+ .

(2) If the sequent $\Gamma \succ \Delta$ is derivable in $\mathrm{LKD}^{+}, \Gamma^{\prime} \succ \Delta^{\prime}$ is derivable in $\mathrm{LKD}$ !.

Proof. (1) holds since $(\square)$ is a special case of $(\square \diamond)$.

(2) follows from repeated application of lemma 2 and theorem 2.3.

\footnotetext{
${ }^{28}$ We thank the anonymous referee for suggesting this notation.
} 
The system LKD! ${ }^{+}$could be used to study the absolutely positive fragment of LKD!, which is obtained by adding primitive $\diamond$ and dropping $~$ and $\supset .{ }^{29}$ We will not, however, investigate the absolutely positive fragment further here.

Before moving on to the quantified modal logic, we will note that LKD! lacks a desirable feature: it does not uniquely define the connective $\square .{ }^{30}$ If another connective, $\circ$, is added with the same rules, the sequents $\square A \succ \circ A$ and $\circ A \succ \square A$ will not be derivable. The closest we come is proving $\square \circ A \succ \square \circ A$ and $\circ \square A \succ \circ \square A$.

What does this mean for LKD!? If we view the rule for $\square$ as settling its meaning, then the system will appear deficient. It would be better to have separate rules for introducing a single box on the left and on the right. It is, however, not clear how to do this in a Gentzen-style sequent system. This problem is intimately related to the problem with the Barcan formula that motivates the hypersequent system, HQD!, as we will see in the next section.

\section{Hypersequent system}

Let us turn to the first-order version of KD!, QD! As mentioned, QD! takes as an axiom scheme the Barcan formula, with the converse Barcan formula following from the other axioms and rules. Perhaps unsurprisingly, in light of the failure of the Hilbert-style system to prove the Barcan formula, the result of adding the quantifier rules,

$$
\frac{A, \Gamma \succ \Delta}{\forall x A, \Gamma \succ \Delta}(\forall \succ) \quad \frac{\Gamma \succ \Delta, A(y)}{\Gamma \succ \Delta, \forall x A(x)}(\succ \forall)
$$

where $y$ cannot occur freely in the conclusion of $(\succ \forall)$, to the sequent system LKD! does not permit a proof of the Barcan formula. It does, however, permit a proof of the converse Barcan formula.

$$
\frac{\frac{A \succ A}{\forall x A \succ A}(\forall \succ)}{\frac{\square \forall x A \succ \square A}{\square}(\square)}(\succ \forall)
$$

\footnotetext{
${ }^{29}$ The term "absolutely positive fragment" comes from Dunn (1995).

${ }^{30}$ The significance of this feature was pointed out by Belnap (1962). For more discussion, see, for example, Wansing (1998) or Restall (2000). We would like to thank Greg Restall for suggesting that we check it in this case.
} 
The problem with deriving the Barcan formula comes from the quantifier on the right.

$$
\frac{\frac{A \succ A}{A \succ \forall x A}}{\frac{\square(?)}{\square \succ \square \forall x A}}{ }^{(\square)}(\forall \succ)
$$

The proof breaks down at the step labelled (??). In order to apply the $(\succ \forall)$ rule, $x$ cannot be free in $A$. We are considering arbitrary $A$ here, so the restriction blocks the proof. We have not been able to adjust the sequent system so that it proves the Barcan formula. ${ }^{31}$ We have, however, formulated a hypersequent system in which the Barcan formula is provable.

In $\S 2$, we noted that the rules lack a desirable property, namely a kind of uniqueness proof showing that the rule $(\square)$ defines the connective $\square$. The problem stems from $(\square)$ introducing the connective on both the left and the right. The natural solution is a pair of rules, one for introducing the connective on the left and one for the right. Such a rule would fix the problem with the derivation of the Barcan formula. We can formulate such rules, but they require adopting a generalization of sequents.

A hypersequent is a non-empty sequence of sequents. ${ }^{32}$

$$
\Gamma_{0} \succ \Delta_{0}|\ldots| \Gamma_{n} \succ \Delta_{n}
$$

The sequents in a hypersequent sequence are its components. A hypersequent with $n$ components is an $n$-hypersequent. We will use the notation $\mathcal{H}[\Gamma \succ \Delta]$ for a hypersequent in which there is a particular occurrence of the component $\Gamma \succ \Delta$. We will use the notation $\mathcal{H}_{n}\left[\Gamma_{k} \succ \Delta_{k}\right]$ to indicate that the hypersequent is an $n$-hypersequent and the displayed component is the $k$ th one. We will also use the notation $\mathcal{H}\left[\Gamma \succ_{k} \Delta\right]$ to indicate that the displayed component is the $k$ th one. The notation $\mathcal{H}\left[\Gamma \succ \Delta \mid \Gamma^{\prime} \succ \Delta^{\prime}\right]$ will be used for a hypersequent with the two indicated occurrences of the displayed components, which are next to each other in that order. We will use $\emptyset_{n}$ for the $n$-hypersequent with all its components empty, with the convention that if $n \leq 0$, then $\emptyset_{n}$ is

\footnotetext{
${ }^{31}$ One idea would be to add additional structural connectives, but this idea quickly begins to look like a hypersequent.

${ }^{32}$ Hypersequents were discovered by G. E. Minc, who called them 'corteges', and independently by Garrel Pottinger and by Arnon Avron, in Minc (1971), Pottinger (1983), and Avron (1987), respectively. Some logicians take hypersequents to be sets or multisets of sequents, rather than sequences. Our notation is based on that of Restall (2012). We thank the anonymous referee and Rohan French for bringing the Minc paper to my attention.
} 
empty. We will abuse notation slightly and use $\mathcal{H} \mid \mathcal{H}^{\prime}$ for the hypersequent obtained by prefixing the components of the $n$-hypersequent $\mathcal{H}^{\prime}$ with the components of the $m$-hypersequent $\mathcal{H}$ to obtain an $(n+m)$-hypersequent. In other words, the first $m$ components of $\mathcal{H} \mid \mathcal{H}^{\prime}$ are the components, in order, of $\mathcal{H}$, followed by the components, in order, of $\mathcal{H}^{\prime}$.

The rules for the system HQD! are as follows. For each $n$, an axiom $n$-hypersequent is an $n$-hypersequent all of whose components are empty except for one, which has an axiom sequent.

$$
\succ|\ldots| p \succ p|\ldots| \succ
$$

The usual truth-functional connectives have left and right rules for each component. In the following, $\Sigma_{i}=\Gamma_{i}, \Gamma_{i}^{\prime}$ and $\Theta_{i}=\Delta_{i}, \Delta_{i}^{\prime}$.

$$
\begin{gathered}
\frac{\mathcal{H}[\Gamma \succ \Delta, A]}{\mathcal{H}[\sim A, \Gamma \succ \Delta]}(\sim \succ) \quad \frac{\mathcal{H}[A, \Gamma \succ \Delta]}{\mathcal{H}[\Gamma \succ \Delta, \sim A]}(\succ \sim) \\
\frac{\Gamma_{0}^{\prime} \succ \Delta_{0}^{\prime}|\ldots| \Gamma_{k}^{\prime} \succ \Delta_{k}^{\prime}, A|\ldots| \Gamma_{n}^{\prime} \succ \Delta_{n}^{\prime} \quad}{\Sigma_{0} \succ \Theta_{0}|\ldots| \Sigma_{k} \succ \Theta_{k}, A \& B|\ldots| \Sigma_{n} \succ \Theta_{n}} \\
\frac{\mathcal{H}[\Gamma, A, B \succ \Delta]}{\mathcal{H}[\Gamma, A \& B \succ \Delta]}(\& \succ) \quad \frac{\mathcal{H}[\Gamma \succ \Delta, A, B]}{\mathcal{H}[\Gamma \succ \Delta, A \vee B]}(\succ \vee) \\
\frac{\Gamma_{0}^{\prime} \succ \Delta_{0}^{\prime}|\ldots| A, \Gamma_{k}^{\prime} \succ \Delta_{k}^{\prime}|\ldots| \Gamma_{n}^{\prime} \succ \Delta_{n}^{\prime} \quad \Gamma_{0} \succ \Delta_{0}|\ldots| B, \Gamma_{k} \succ \Delta_{k}|\ldots| \Gamma_{n} \succ \Delta_{n}}{\Sigma_{0} \succ \Theta_{0}|\ldots| A \vee B, \Sigma_{k} \succ \Theta_{k}|\ldots| \Sigma_{n} \succ \Theta_{n}}(\vee \succ) \\
\frac{\Gamma_{0}^{\prime} \succ \Delta_{0}^{\prime}|\ldots| \Gamma_{k}^{\prime} \succ \Delta_{k}^{\prime}, A|\ldots| \Gamma_{n}^{\prime} \succ \Delta_{n}^{\prime} \quad \Gamma_{0} \succ \Delta_{0}|\ldots| B, \Gamma_{k} \succ \Delta_{k}|\ldots| \Gamma_{n} \succ \Delta_{n}}{\Sigma_{0} \succ \Theta_{0}|\ldots| A \supset B, \Sigma_{k} \succ \Theta_{k}|\ldots| \Sigma_{n} \succ \Theta_{n}}(\supset \succ) \\
\frac{\mathcal{H}[\Gamma, A \succ \Delta, B]}{\mathcal{H}[\Gamma \succ \Delta, A \supset B]}(\succ \supset)
\end{gathered}
$$

Each component has the full set of structural rules, but there are no structural rules for manipulating the structure of hypersequents.

The quantifier rules are as follows.

$$
\frac{\mathcal{H}[A, \Gamma \succ \Delta]}{\mathcal{H}[\forall x A, \Gamma \succ \Delta]}(\forall \succ) \quad \frac{\mathcal{H}[\Gamma \succ \Delta, A(y)]}{\mathcal{H}[\Gamma \succ \Delta, \forall x A(x)]}(\succ \forall)
$$

In the $(\succ \forall)$ rule, $y$ cannot occur freely in the conclusion hypersequent. The modal rules are the following.

$$
\frac{\mathcal{H}\left[\Gamma \succ \Delta \mid A, \Gamma^{\prime} \succ \Delta^{\prime}\right]}{\mathcal{H}\left[\square A, \Gamma \succ \Delta \mid \Gamma^{\prime} \succ \Delta^{\prime}\right]}(\square \succ) \quad \frac{\mathcal{H}\left[\Gamma \succ \Delta \mid \Gamma^{\prime} \succ \Delta^{\prime}, A\right]}{\mathcal{H}\left[\Gamma \succ \Delta, \square A \mid \Gamma^{\prime} \succ \Delta^{\prime}\right]}(\succ \square)
$$


As mentioned, there are no structural rules for manipulating the hypersequents. ${ }^{33}$ This renders the hypersequents non-commutative. The hypersequents can be seen as a notational variant of 2 -sequents, reading hypersequents left to right as 2-sequents top to bottom. ${ }^{34}$ With this in mind, the 2 -sequent rule $(\succ \square)$ is, in our notation, the following, where the displayed components are rightmost.

$$
\frac{\mathcal{H}[\Gamma \succ \Delta \mid \succ A]}{\mathcal{H}[\Gamma \succ \Delta, \square A \mid \succ]}(\succ \square)
$$

The system of 2-sequents, as presented in Masini (1992), is a system for the modal logic KD. Models for KD need not have functional accessibility relations, so introducing $\square$ on the right needs to have a degree of generality not found in our rule $(>\square)$. Conversely, one could adapt the 2 -sequents to cover KD! by strengthening the rule.

The cut rule for HQD! is the following.

$\frac{\Gamma_{0}^{\prime} \succ \Delta_{0}^{\prime}|\ldots| \Gamma_{k}^{\prime} \succ \Delta_{k}^{\prime}, A|\ldots| \Gamma_{n}^{\prime} \succ \Delta_{n}^{\prime} \quad \Gamma_{0} \succ \Delta_{0}|\ldots| A, \Gamma_{k} \succ \Delta_{k}|\ldots| \Gamma_{n} \succ \Delta_{n}}{\Sigma_{0} \succ \Theta_{0}|\ldots| \Sigma_{k} \succ \Theta_{k}|\ldots| \Sigma_{n} \succ \Theta_{n}}$ (cut)

The system HQD! does not have cut among its basic rules, but we can show that it is admissible. The strategy here is similar to the strategy for the proof of the Elimination Theorem for LKD!. We show that a stronger rule,

$\frac{\Gamma_{0}^{\prime} \succ \Delta_{0}^{\prime}|\ldots| \Gamma_{k}^{\prime} \succ \Delta_{k}^{\prime}, A|\ldots| \Gamma_{n}^{\prime} \succ \Delta_{n}^{\prime} \quad \Gamma_{0} \succ \Delta_{0}|\ldots| A, \Gamma_{k} \succ \Delta_{k}|\ldots| \Gamma_{n} \succ \Delta_{n}}{\Sigma_{0} \succ \Theta_{0}|\ldots| \Gamma_{k}^{-A}, \Gamma_{k}^{\prime} \succ \Delta_{k}, \Delta_{k}^{\prime-A}|\ldots| \Sigma_{n} \succ \Theta_{n}}$ (mix)

is admissible. The mix formula is the displayed formula $A$, in the displayed component in the mix. The proof proceeds via a double induction on grade and rank, as before, with a change to the definition of rank. The left rank of a mix is the length of the longest upward path beginning at the left premiss of the mix containing an occurrence of the mix formula in the indicated component to the right of the turnstile. The right rank is defined similarly. The rank of a mix is the sum of the left and right ranks. Intuitively, the rank of the mix is calculated with respect to the particular component. The

\footnotetext{
${ }^{33}$ The hypersequent system of Indrzejczak (2016) explores linear temporal logic using sequences of sequents with no external structural rules. Indrzejczak's modal rules are, however, different. It would be interesting to see how the modal rules of HQD! can be combined with Indrzejczak's.

${ }^{34}$ For more on 2-sequents, see Masini (1992) and Lellmann (2015). We would like to thank the anonymous referee for drawing my attention to 2-sequents.
} 
proof strategy is to permute mix above rules, reducing the left rank, and then the right rank, and finally reducing the grade, with the usual special cases for weakening and axioms.

TheOREM 3.1 (Mix elimination for HQD!). The cut rule is admissible for HQD!

Proof. For cases in which the mix formula is in the component in which a classical connective is being introduced or a structural rule is being applied, then the case is handled in the same way as in the Gentzen-style sequent calculus of the previous section. We will do a few cases to illustrate. The cases in which the rules affect formulas in a component that does not contain the mix formula are immediate.

Suppose that the rank of the mix formula is 2 and it is of the form $A \& B$. We will, throughout the proof, use the notation $\mathcal{H}^{i}$ for the wider context of the different premiss and conclusion sequents, and $\Sigma$ and $\Theta$ will be the union of the relevant $\Gamma$ and $\Delta$.

$$
\frac{\frac{\mathcal{H}^{1}[\Gamma \succ \Delta, A] \quad \mathcal{H}^{2}\left[\Gamma^{\prime} \succ \Delta^{\prime}, B\right]}{\mathcal{H}^{3}\left[\Gamma, \Gamma^{\prime} \succ \Delta, \Delta^{\prime}, A \& B\right]}(\succ \&) \frac{\mathcal{H}^{4}\left[\Gamma^{\prime \prime}, A, B \succ \Delta^{\prime \prime}\right]}{\mathcal{H}^{4}\left[\Gamma^{\prime \prime}, A \& B \succ \Delta^{\prime \prime}\right]}}{\mathcal{H}^{5}[\Sigma \succ \Theta]}(\& \succ)
$$

This case is handled by permuting the mix above the connective rules as follows.

$$
\frac{\mathcal{H}^{2}\left[\Gamma^{\prime} \succ \Delta^{\prime}, B\right]}{\frac{\mathcal{H}^{1}[\Gamma \succ \Delta, A] \quad \mathcal{H}^{4}\left[\Gamma^{\prime \prime}, A, B \succ \Delta^{\prime \prime}\right]}{\mathcal{H}^{6}\left[\Gamma, \Gamma^{\prime \prime-A}, B \succ \Delta^{-A}, \Delta^{\prime \prime}\right]}(\operatorname{mix})} \underset{\text { mix })}{\mathcal{H}^{5}\left[\Sigma^{\prime} \succ \Theta^{\prime}\right]}
$$

Both mixes can be eliminated by the inductive hypothesis on grade, which has decreased. In the conclusion hypersequent, $\Sigma^{\prime}$ and $\Theta^{\prime}$ are $\Sigma$ and $\Theta$, respectively, except possibly that the former are missing some occurrences of $A$ and $B$ in the latter. These can be replaced using the weakening rules as required to obtain the desired hypersequent.

As another example, suppose that the right rank of the mix is greater than 1 , the left rank is 1 , and the mix formula is not $A \& B$, then the case looks like the following.

$$
\frac{\mathcal{H}^{1}[\Gamma \succ \Delta] \frac{\mathcal{H}^{2}\left[\Gamma^{\prime}, A, B \succ \Delta^{\prime}\right]}{\mathcal{H}^{2}\left[\Gamma^{\prime}, A \& B \succ \Delta^{\prime}\right]}}{\mathcal{H}^{3}[\Sigma, A \& B \succ \Theta]}(\text { mix })
$$


The mix is permuted upwards as follows.

$$
\frac{\mathcal{H}^{1}[\Gamma \succ \Delta] \mathcal{H}^{2}\left[\Gamma^{\prime}, A, B \succ \Delta^{\prime}\right]}{\frac{\mathcal{H}^{3}[\Sigma, A, B \succ \Theta]}{\mathcal{H}^{3}[\Sigma, A \& B \succ \Theta]}(\& \succ)}(\operatorname{mix})
$$

If the mix formula was $A$ or $B$, then an additional weakening step is inserted preceding the final $(\& \succ)$.

Suppose that the case is as before except that the mix formula is of the form $A \& B$, which occurs in $\Delta$. Then the case is handled as in the previous example, except that the derivation is extended with the following.

$$
\frac{\mathcal{H}^{1}[\Gamma \succ \Delta] \quad \mathcal{H}^{3}\left[\Gamma^{\prime}, A \& B \succ \Delta^{\prime}\right]}{\mathcal{H}^{4}\left[\Sigma^{-A \& B}, \Gamma \succ \Delta^{-A \& B}, \Theta^{\prime}\right]}(\operatorname{mix})
$$

As $\Gamma$ is a subset of $\Sigma$, and $\Delta^{-A \& B}$ is a subset of $\Theta$, the desired concluding hypersequent can be obtained via applications of contraction. Apart from the wider hypersequent context, the classical connective cases have followed the mould of the proof for the classical sequent system. The cases in which the left rank is greater than 1 are handled in much the same way.

The important new cases are the ones involving the $\square$ rules. First is the case in which the mix formula is $\square A$ and the mix formula was just introduced on both sides, i.e. the rank is 2 .

$\frac{\frac{\mathcal{H}^{1}\left[\Gamma_{k}^{\prime} \succ \Delta_{k}^{\prime} \mid \Gamma_{k+1}^{\prime} \succ \Delta_{k+1}^{\prime}, A\right]}{\mathcal{H}^{1}\left[\Gamma_{k}^{\prime} \succ \Delta_{k}^{\prime}, \square A \mid \Gamma_{k+1}^{\prime} \succ \Delta_{k+1}^{\prime}\right]}(\succ \square) \frac{\mathcal{H}^{2}\left[\Gamma_{k} \succ \Delta_{k} \mid A, \Gamma_{k+1} \succ \Delta_{k+1}\right]}{\mathcal{H}^{2}\left[\square A, \Gamma_{k} \succ \Delta_{k} \mid \Gamma_{k+1} \succ \Delta_{k+1}\right]}}{\mathcal{H}^{3}\left[\Sigma_{k} \succ \Theta_{k} \mid \Sigma_{k+1} \succ \Theta_{k+1}\right]}$ (mix)

Since the rank is 2 , the mix formula does not otherwise occur in $\Delta_{k}^{\prime}$ or $\Gamma_{k}$.

The mix can then be carried out on simpler formulas in the following way.

$$
\frac{\mathcal{H}^{1}\left[\Gamma_{k}^{\prime} \succ \Delta_{k}^{\prime} \mid \Gamma_{k+1}^{\prime} \succ \Delta_{k+1}^{\prime}, A\right] \quad \mathcal{H}^{2}\left[\Gamma_{k} \succ \Delta_{k} \mid A, \Gamma_{k+1} \succ \Delta_{k+1}\right]}{\mathcal{H}^{3}\left[\Sigma_{k} \succ \Theta_{k} \mid \Gamma_{k+1}^{\prime}, \Gamma_{k+1}^{-A} \succ \Delta_{k+1}, \Delta_{k+1}^{\prime-A}\right]}(\operatorname{mix})
$$

The $(K \succ)$ and $(\succ K)$ rules can then be used to obtain the desired end hypersequent.

The second important new case is the one in which the left rank of the mix formula is greater than 1 , and the rule leading to the left premiss is 
$(\succ \square)$. This case splits into two subcases, depending on whether $\square A \in \Delta_{k}$. For the first subcase, assume $\square A \notin \Delta_{k}$.

$$
\frac{\frac{\mathcal{H}^{1}\left[\Gamma_{k}^{\prime} \succ \Delta_{k}^{\prime} \mid \Gamma_{k+1}^{\prime} \succ \Delta_{k+1}^{\prime}, A\right]}{\mathcal{H}^{1}\left[\Gamma_{k}^{\prime} \succ \Delta_{k}^{\prime}, \square A \mid \Gamma_{k+1}^{\prime} \succ \Delta_{k+1}^{\prime}\right]}(\succ \square) \quad \mathcal{H}^{2}\left[\square A, \Gamma_{k} \succ \Delta_{k}\right]}{\mathcal{H}^{3}\left[\Gamma_{k}^{-\square A}, \Gamma_{k}^{\prime} \succ \Delta_{k}, \Delta_{k}^{\prime-\square A} \mid \Sigma_{k+1} \succ \Theta_{k+1}\right]} \text { (mix) }
$$

Since the left rank of the mix formula is greater than 1 , we can eliminate the mixes in following way. Since $\left(\Gamma^{-\square A}\right)^{-\square A}=\Gamma^{-\square A}$, we will write the former as the latter.

$$
\frac{\frac{\mathcal{H}^{1}\left[\Gamma_{k}^{\prime} \succ \Delta_{k}^{\prime} \mid \Gamma_{k+1}^{\prime} \succ \Delta_{k+1}^{\prime}, A\right] \quad \mathcal{H}^{2}\left[\square A, \Gamma_{k} \succ \Delta_{k}\right]}{\mathcal{H}^{3}\left[\Gamma_{k}^{-\square A}, \Gamma_{k}^{\prime} \succ \Delta_{k}, \Delta_{k}^{\prime-\square A} \mid \Sigma_{k+1} \succ \Theta_{k+1}, A\right]}(\operatorname{mix})}{\frac{\mathcal{H}^{3}\left[\Gamma_{k}^{-\square A}, \Gamma_{k}^{\prime} \succ \Delta_{k}, \Delta_{k}^{\prime-\square A}, \square A \mid \Sigma_{k+1} \succ \Theta_{k+1}\right]}{\mathcal{H}^{4}\left[\Gamma_{k}^{-\square A}, \Gamma_{k}^{-\square A}, \Gamma_{k}^{\prime} \succ \Delta_{k}, \Delta_{k}^{-\square A}, \Delta_{k}^{\prime-\square A}\right]} \mathcal{H}^{2}\left[\square A, \Gamma_{k} \succ \Delta_{k}\right]} \text { (mix) }
$$

The topmost mix can be eliminated via the induction hypothesis on rank, since it has a lower rank than the original mix. The bottommost mix can be eliminated by the same induction hypothesis, since its rank is lower than the original rank, as the left rank is 1 and the right rank is equal to the original right rank. The desired end hypersequent can then be obtained by using the contraction rules to eliminate the extra copies of formulas from the second mix.

For the second subcase, assume $\square A \in \Delta_{k}$.

$$
\frac{\frac{\mathcal{H}^{1}\left[\Gamma_{k}^{\prime} \succ \Delta_{k}^{\prime} \mid \Gamma_{k+1}^{\prime} \succ \Delta_{k+1}^{\prime}, A\right]}{\mathcal{H}^{1}\left[\Gamma_{k}^{\prime} \succ \Delta_{k}^{\prime}, \square A \mid \Gamma_{k+1}^{\prime} \succ \Delta_{k+1}^{\prime}\right]}(\succ \square) \quad \mathcal{H}^{2}\left[\square A, \Gamma_{k} \succ \Delta_{k}\right]}{\mathcal{H}^{3}\left[\Gamma_{k}^{-\square A}, \Gamma_{k}^{\prime} \succ \Delta_{k}, \Delta_{k}^{\prime-\square A} \mid \Sigma_{k+1} \succ \Theta_{k+1}\right]}(\operatorname{mix})
$$

This is transformed into the following.

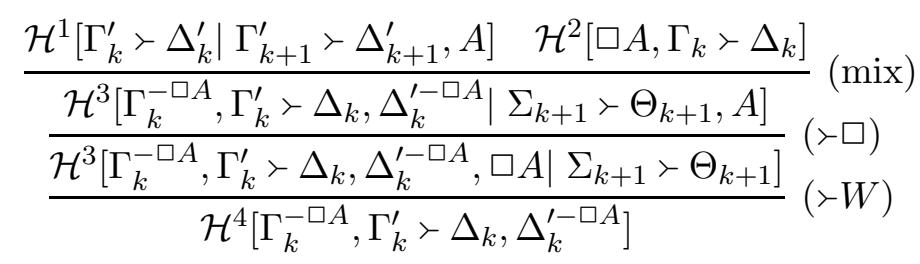

The last step is justified because $\square A \in \Delta_{k}$. The displayed $\square A$ is contracted into a copy in $\Delta_{k}$, obtaining the desired endsequent.

The case in which the left rank is 1 and the right rank is greater than 1 is handled in much the same way as the previous case, so we omit it. 
The cases in which the mix formula is of the form $\square A$ and is obtained via one of the structural rules are similar to the classical cases, so we omit them.

Given contraction, the following, simpler cut rule is admissible.

$$
\frac{\mathcal{H}_{n}\left[\Gamma \succ_{k} \Delta, A\right] \quad \mathcal{H}_{n}\left[A, \Gamma \succ_{k} \Delta\right]}{\mathcal{H}_{n}\left[\Gamma \succ_{k} \Delta\right]}\left(\mathrm{cut}^{\prime}\right)
$$

There are three features of HQD! we will note. The first concerns an external form of weakening. The height of a proof is the length of any of the longest paths from the end hypersequent to an axiom. We will say that a rule is height-preserving admissible just in case, if there is a derivation of the premises that has height $n$, then there is a derivation of the conclusion that has height at most $n$.

LEMma 3. The rules

$$
\frac{\mathcal{H}}{\succ \mid \mathcal{H}} \quad \frac{\mathcal{H}}{\mathcal{H} \mid \succ}
$$

are height-preserving admissible.

Proof. We will sketch the proof for one, since the proof for the other is similar. The proof proceeds by induction on the height of the derivation. For a given proof of $\mathcal{H}_{n}$, replace all axioms in the derivation with the corresponding $(n+1)$-hypersequent axiom, with the position of the formulas determined by counting from the right. The proof can be carried out using the same inference rules as before to obtain $\succ \mid \mathcal{H}$.

Height-preservation follows from the fact that we only change the number of components in the axioms, not any of the rules used. Thus the height is preserved.

This immediately gives the following two corollaries.

Corollary 1. Let $\mathcal{H}$ be a hypersequent. The rules

$$
\frac{\mathcal{H}}{\Gamma \succ \Delta \mid \mathcal{H}} \quad \frac{\mathcal{H}}{\mathcal{H} \mid \Gamma \succ \Delta}
$$

are admissible.

Proof. Apply lemma 3 and then use repeated instances of the $K$ rules to obtain the desired end hypersequent. 
The following corollary of lemma 3 will be used the proof of theorem 3.3 below. Let us say that $A$ is an $n$-theorem iff $\mathcal{H}_{n}\left[\succ_{0} A\right]$ is derivable where the wider context is empty.

Corollary 2. If $A$ is an $n$-theorem, then, $A$ is an $(n+m)$-theorem, for all $m \geq 0$.

The second notable feature of HQD! is that a standard theorem for sequent systems, the identity theorem, fails for it. The identity theorem says that $B \succ B$ is derivable, for arbitrary $B$. This is particularly interesting in systems whose axioms are atomic, $p \succ p$, such as ours. The identity theorem fails for HQD!, since $\square A \succ \square A$ is not derivable for arbitrary formulas $A$. Note, however, that a slightly weakened form is provable. Let the modal depth, $d(A)$, of $A$ be the greatest number of nested boxes in $A$.

Theorem 3.2. If $d(A) \leq n$, then $A \succ A \mid \emptyset_{n}$ is derivable.

Proof. The proof is by a double induction on the modal depth and complexity of $A$.

Assume that for all formulas $B$ such that $d(B)<n, B \succ B \mid \emptyset_{n-1}$ is derivable. We now proceed by induction on the complexity of $A$.

If $A$ is of the form $\square B$, then we have the following derivation, whose first line is justified by assumption and whose second is justified by lemma 3 .

$$
\frac{\frac{B \succ B \mid \emptyset_{n-1}}{\succ|B \succ B| \emptyset_{n-1}}}{\frac{\succ \square|B \succ| \emptyset_{n-1}}{\square B \succ \square B|\succ| \emptyset_{n-1}}(\ulcorner\succ)}(\square)
$$

We note that if $d(B)<n$, then by the induction hypothesis, $B>B \mid \emptyset_{n-1}$ is derivable, whence corollary 2 yields $B \succ B \mid \emptyset_{n}$. The remaining cases are taken care of by the induction hypothesis on complexity.

A third interesting feature of HQD!, which is the main reason we investigate it, is that it permits derivations of the instances of the Barcan formula, unlike LKD! with the quantifier rules. Here is a proof of the Barcan formula, given theorem 3.2 and lemma 3 .

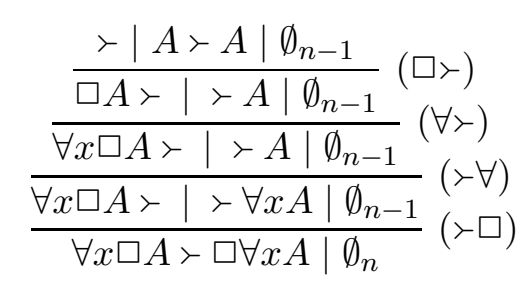


HQD! is equivalent to QD! in the following way. Define a function $f$ on hypersequents $\Gamma_{0} \succ \Delta_{0}|\ldots| \Gamma_{n} \succ \Delta_{n}$ as

$$
f\left(\Gamma_{0} \succ \Delta_{0}|\ldots| \Gamma_{n} \succ \Delta_{n}\right)=\left(\bigwedge \Gamma_{0} \supset \bigvee \Delta_{0}\right) \vee \square\left(f\left(\Gamma_{1} \succ \Delta_{1}|\ldots| \Gamma_{n} \succ \Delta_{n}\right)\right) .{ }^{35}
$$

Before stating and proving the equivalence theorems, we will prove one required lemma concerning an unusual theorem of KD!.

LEMmA 4. The sentence

$$
((A \supset B) \vee \square(C \& D \supset E)) \supset((A \& \square C \supset B) \vee \square(D \supset E))
$$

is derivable in $\mathrm{KD}$ !.

Proof. We provide a proof in LKD!, omitting a few structural steps for brevity.

$$
\begin{aligned}
& \frac{A \supset B, A \succ B}{A \supset B, A \& \square C \succ B}(\& \succ) \quad \frac{C \& D \supset E, C \succ D \supset E}{\square(C \& D \supset E), \square C \succ \square(D \supset E)}(\square) \\
& \frac{(A \supset B) \vee \square(C \& D \supset E), A \& \square C \succ B, \square(D \supset E)}{(A \supset B) \vee \square(C \& D \supset E) \succ A \& \square C \supset B, \square(D \supset E)}(\succ \supset) \\
& \frac{(A \supset B) \vee \square(C \& D \supset E) \succ(A \& \square C \supset B) \vee \square(D \supset E)}{(\succ \vee)}
\end{aligned}
$$

The desired conclusion then follows from theorem 2.2.

Define $\square^{k}$ by recursion as follows: $\square^{0} A=A$ and $\square^{n+1} A=\square\left(\square^{n} A\right)$. We can now prove the equivalence of QD! and HQD!

\section{Theorem 3.3.}

1. If $\mathrm{QD} ! \vdash A$, then for some $n, A$ is an $n$-theorem of $\mathrm{HQD}$ !

2. If $\mathcal{H}$ is derivable in $\mathrm{HQD}$ !, then $\mathrm{QD} ! \vdash f(\mathcal{H})$.

Proof. The proof of (1) proceeds by an adaptation of a standard inductive argument. The only wrinkle is that one must take into account the modal depth of the sentences involved. We will present just the case for the rule of modus ponens.

The modus ponens case is handled using corollary 2. Suppose that $A$ is an $n$-theorem and $A \supset B$ is an $m$-theorem. Let $k=\max (n, m)$. Then, by corollary 2 , both are $k$-theorems. There is a proof of

$$
\succ A \supset B \mid \emptyset_{k-1}
$$

\footnotetext{
${ }^{35}$ If $\Gamma=A_{1}, \ldots, A_{n}$, then $\bigwedge \Gamma=A_{1} \& \ldots \& A_{n}$ and $\bigvee \Gamma=A_{1} \vee \ldots \vee A_{n}$. If $\Gamma=\emptyset$, then $\bigwedge \Gamma=\top$, and $\bigvee \Gamma=\perp$.
} 
but, by the elimination theorem,

$$
A \succ B \mid \emptyset_{k-1}
$$

is derivable. By the elimination theorem again, there is then a proof of

$$
\succ B \mid \emptyset_{k-1}
$$

which is the desired conclusion.

The proof of (2) proceeds by inductively showing that the translations of the axioms of HQD! are derivable in QD! and that if the translations of the premises of a rule are derivable in QD! then so is the translation of the conclusion. There are many cases to check, so we will present a sketch of the more interesting ones here. First, an axiom case. The axioms are hypersequents of the form

$$
\mathcal{H}\left[p \succ_{k} p\right],
$$

where the undisplayed components are all empty. The translation of this hypersequent is equivalent to $\square^{k}(p \supset p)$, which is an QD! theorem.

For the $(\square \succ)$ case, assume that $\mathcal{H}\left[\Gamma_{k} \succ \Delta_{k} \mid \Gamma_{k+1}, A \succ \Delta_{k+1}\right]$ is derivable in HQD!. We want to show that the translation of $\mathcal{H}\left[\Gamma_{k}, \square A \succ \Delta_{k} \mid \Gamma_{k+1} \succ \Delta_{k+1}\right]$ is $\mathrm{QD}$ ! derivable. By the induction hypothesis, the translation of the premiss is derivable in QD!, so the conclusion is derivable using lemma 4 and some propositional equivalences and the modal axioms.

For the $\succ \forall$ case, assume that $\mathcal{H}\left[\Gamma_{k} \succ \Delta_{k}, A\right]$ is derivable in HQD! and that the translation is QD! derivable. We want to show that the translation of $\mathcal{H}\left[\Gamma_{k} \succ \Delta_{k}, \forall x A\right]$, where $x$ is not free in $\mathcal{H}$, is QD! derivable. Using lemma 1 , the translation of the premiss can be shown equivalent to $X \vee \square^{k}\left(\bigwedge \Gamma_{k} \supset\right.$ $\left.\vee \Delta_{k} \vee A\right) \vee Y$, for some formulas $X$ and $Y$. By assumption this is QD! derivable. By the rule of generalization,

$$
\forall x\left(X \vee \square^{k}\left(\bigwedge \Gamma_{k} \supset \bigvee \Delta_{k} \vee A\right) \vee Y\right)
$$

is QD! derivable. Since $x$ is not free in $\mathcal{H}\left[\Gamma_{k} \succ \Delta_{k}, \forall x A\right], x$ is not free in $\mathcal{H}\left[\Gamma_{k} \succ \Delta_{k}, A\right]$, except possibly in the displayed occurrence of $A$. Therefore, by use of the Barcan formula and the logical truth $\forall x(D \vee E) \supset(D \vee \forall x E)$, where $x$ is not free in $D$,

$$
\forall x\left(X \vee \square^{k}\left(\bigwedge \Gamma_{k} \supset \bigvee \Delta_{k} \vee A\right) \vee Y\right)
$$

implies

$$
X \vee \square^{k} \forall x\left(\bigwedge \Gamma_{k} \supset \bigvee \Delta_{k} \vee A\right) \vee Y
$$


From this we obtain

$$
X \vee \square^{k}\left(\bigwedge \Gamma_{k} \supset \bigvee \Delta_{k} \vee \forall x A\right) \vee Y
$$

which was to be shown.

We now return to KD! and QD! for the rest of the paper.

\section{Features of KD! and QD!}

Having presented alternative proof systems for KD! and QD!, we will prove some theorems concerning those logics. First, we show that the set of tautologies for KD! and the set of logical truths for QD! reduce to classical tautologies and first-order logical truth, respectively. Then, in the next section we bring out a connection between QD! and a proof system for circular definitions in revision theory.

We now define a reduction relation, $\triangleright$, on pairs of sentences, or formulas, in the language of KD!, or QD!, respectively. Roughly, $A \triangleright^{*} A^{\prime}$ iff $A^{\prime}$ is the result of distributing some box in $A$ one level in. More precisely, say $A \triangleright^{*} B$ just in case $B$ is just like $A$ except that where $A$ has one occurrence of a subformula in the left-hand column, $B$ has the corresponding subformula in the right-hand column.

\begin{tabular}{ll}
$A$ & $B$ \\
\hline$\square \sim C$ & $\sim \square C$ \\
$\square \forall x C$ & $\forall x \square C$ \\
$\square(C \& D)$ & $\square C \& \square D$ \\
$\square(C \vee D)$ & $\square C \vee \square D$ \\
$\square(C \supset D)$ & $\square C \supset \square D$
\end{tabular}

Let $\triangleright$ be the reflexive, transitive closure of $\triangleright^{*}$.

Let us define box normal form, or bnf, of $A$ as a wff $B$ such that $A \triangleright B$ and for all $C$, if $B \triangleright C$, then $B=C$. Every formula has a bnf, which is unique, although we here omit the proof. We will also note that if $A$ is in bnf, then the bnf of $\square A$ is $A$ with an additional box in front of every occurrence of each atom in $A$.

We will need some terminology. Let us say a formula $A$ is a boxed atom iff for some $n \geq 0$, it has the form $\square^{n} p$, where $p$ is an atom. A maximal boxed atom is an occurrence of a boxed atom that is not a proper subformula 
occurrence of another boxed atom occurrence. As an example, in the sentence $\square^{4} p \& \square^{2} p \& p$ there are three maximal boxed atoms, $\square^{4} p, \square^{2} p$, and $p$, although both of the latter two also occur as subformulas of the first. We will restrict attention to maximal boxed atoms.

For the following proofs, we will use the notation $A^{\prime}$ for the bnf of $A$. We will also use tautology, and first-order logical truth, to mean, respectively, a substitution instance of a classical tautology, or first-order logical truth, in the language of KD!, or QD!, respectively. To be a bit more precise in the first-order case, a substitution $s$ is a function from formulas to formulas such that

- $s(A * B)=s(A) * s(B)$, for $* \in\{\&, \vee, \supset\}$,

$\bullet s(\bullet A)=\bullet s(A)$, for $\bullet \in\{\square, \forall x, \sim\}$, and

- $s\left(F\left(x_{1}, \ldots, x_{n}\right)\right)=C\left[x_{1} / y_{1}, \ldots, x_{n} / y_{n}\right]$, where $F\left(x_{1}, \ldots, x_{n}\right)$ is an atomic formula and $C$ is a formula with exactly the variables $y_{1}, \ldots, y_{n}$ free, and $\left[x_{1} / y_{1}, \ldots, x_{n} / y_{n}\right]$ is the simultaneous substitution of $x_{i}$ for $y_{i}$, relabeling bound variables to avoid clashes, as required.

A sentence $B$ in the language, with $\square$, is a first-order logical truth iff there is a sentence $A$, in the $\square$-free language, and substitution $s$ such that $B=s(A)$, and $A$ is a first-order logical truth.

Theorem 4.1. For all sentences $A, \mathrm{KD} ! \vdash A$ iff $A^{\prime}$ is a tautology.

Proof. The right-to-left direction follows from repeated use of lemma 1. The proof of the converse is by induction on the proof of $A$. We will present three cases.

The special KD! axiom is $\sim \square A \equiv \square \sim A$. By definition,

$$
\sim \square A \equiv \square \sim A \triangleright^{*} \sim \square A \equiv \sim \square A .
$$

Let $(\square A)^{\prime}$ be $B$. Then the bnf of $\sim \square A \equiv \square \sim A$ is $\sim B \equiv \sim B$, which is a tautology.

The $\mathrm{K}$ axiom is $\square(A \supset B) \supset(\square A \supset \square B)$. By definition,

$$
\square(A \supset B) \supset(\square A \supset \square B) \triangleright^{*}(\square A \supset \square B) \supset(\square A \supset \square B) .
$$

Let $C$ be the bnf of $\square A \supset \square B$. Then the bnf of $\square(A \supset B) \supset(\square A \supset \square B)$ is $C \supset C$, which is a tautology.

Inductive case: Necessitation. Suppose that KD! $\vdash A$. Then, by the induction hypothesis, $A$ has a bnf $A^{\prime}$ that is a tautology. By Necessitation, 
KD! $\vdash \square A$. The bnf of $\square A$ is $A^{\prime}$ with one additional box added to each boxed atom. Since $A^{\prime}$ is a tautology, so is $(\square A)^{\prime}$, which completes this step.

We conclude that for all $A, \mathrm{KD} ! \vdash A$ iff $A^{\prime}$ is a tautology.

TheOrem 4.2. For all formulas $A, \mathrm{QD} ! \vdash A$ iff $A^{\prime}$ is a first-order logical truth.

Proof. As before, the right-to-left direction is straightforward, using lemma 1 and the Barcan formulas. The proof of the converse is by induction on the proof of $A$. It is largely similar to the preceding proof, with additional steps for the quantifier axioms, the Barcan formula, and the rule of Generalization. These additional steps are straightforward, so we omit them.

That is the final result concerning QD! in isolation. We now turn to some connections between QD! and revision theory.

\section{Connections to revision theory}

In this penultimate section, we will connect QD! to the revision theory of circular definitions. We will briefly present the intuitions and ideas behind revision theory. The reader who is familiar with revision theory can skip the next subsection. ${ }^{36}$

\subsection{Revision theory}

Revision theory is a general theory of definitions, including circular definitions. The principle semantic idea behind revision theory is that the meaning of a defined predicate is a revision operator, which is an operation that determines how to revise a hypothetical extension of a defined predicate. One begins with a first-order language, which is expanded with a set of definitions. A set of definitions $\mathscr{D}$ can be any countable set of definitional clauses, although here we will focus on finite sets of definitions with unary definienda, to ease exposition. ${ }^{37}$ The form of definitions we will consider is illustrated in table 5.1. In these clauses, any of the $G_{i}$ can appear in any of the $A_{G_{j}}$.

\footnotetext{
${ }^{36}$ The interested reader should see Gupta and Belnap (1993) for a detailed exposition of basic revision theory. See Standefer (2015) or Gupta and Standefer (2016) for more on extended revision theory.

${ }^{37}$ We will require that only the variables free in the definienda appear freely in the definientia.
} 


$$
\begin{array}{rcc}
G_{1}(x) & ={ }_{D f} & A_{G_{1}}(x) \\
G_{2}(x) & ={ }_{D f} & A_{G_{2}}(x) \\
& \vdots & \\
G_{k}(x) & ={ }_{D f} & A_{G_{k}}(x)
\end{array}
$$

Table 1. Sets of definitions

The base language is interpreted via a classical model $M$, and a $h y$ pothesis, $h$, provides the interpretation of the defined predicate. The set of definitions $\mathscr{D}$ determines a revision operator, $\Delta_{\mathscr{D}, M}$, for each model. Given a model $M$ and a hypothesis $h$, the new hypothesis, $\Delta_{\mathscr{D}, M}(h)$ contains exactly the objects that satisfy $A_{G_{i}}(x)$ according to $M$ and $h$. Define $\Delta_{\mathscr{D}, M}^{0}(h)=h$ and $\Delta_{\mathscr{D}, M}^{n+1}(h)=\Delta_{\mathscr{D}, M}\left(\Delta_{\mathscr{D}, M}^{n}(h)\right)$. The revision operator can be iterated to form revision sequences.

$$
h, \Delta_{\mathscr{D}, M}(h), \Delta_{\mathscr{D}, M}\left(\Delta_{\mathscr{D}, M}(h)\right), \Delta_{\mathscr{D}, M}^{3}(h), \ldots
$$

The logic of circular definitions is defined in terms of these iterations. A sentence $A$ is valid on $\mathscr{D}$ iff for classical models $M$, there is a natural number $p$, such that for all hypotheses $h$, for all $m \geq p, A$ is true according to $M$ and $\Delta_{\mathscr{D}, M}^{m}(h) .{ }^{38}$ A sentence is valid just in case it eventually stabilizes to true, in the sense of reaching a point after which it is only true, in all $\omega$-long revision sequences.

So far we have described basic revision theory, but we need to enrich it to make the connection with QD! clear. One can extend revision theory with a unary operator, $\square .{ }^{39}$ In the extended revision theory, one begins with a $\square$ free language, and adds $\square$ to the expanded language along with the defined predicates. Hypotheses must be extended to settle what objects satisfy a formula of the form $\square A$. After this is done, $\square A$ will be true according to $M$ and $h$, just in case $A$ is true according to $h$. Bringing in revision, $\square A$ is true according to $M$ and $\Delta_{\mathscr{D}, M}(h)$ just in case $A$ is true according to $M$ and $h$.

There are no restrictions on the initial hypotheses, so initially $\square$ may be highly non-compositional. For example, $\square(p \vee \sim p)$ may be false under some hypotheses. As one proceeds along a revision sequence, the $\square$ operator becomes better behaved, so that, e.g., $\square(p \vee \sim p)$ is bound to be true after one revision and $\square \square(p \vee \sim p)$ after two. In fact, the revision-theoretic box

\footnotetext{
${ }^{38}$ This is the $S_{0}$ definition of validity in Gupta and Belnap (1993).

${ }^{39}$ This was done by Standefer (2015) and, in another way, by Gupta and Standefer (2016).
} 
obeys QD! in the following sense: Each of the QD! theorems will be valid, in revision theory, if the modal operator is interpreted as the $\square$ of revision theory.

The revision sequences provide the semantic intuition for why the $\square$ of revision theory might line up with the modal operator. The revision sequences are generated by iterating the revision operator. One can think of these sequences as being Kripke models whose accessibility relation is the predecessor relation on the hypotheses in the sequences, or more accurately pairs comprising the base model and hypotheses from the sequences. The truth condition for the revision-theoretic box can be written as

$$
M, \Delta(h) \Vdash \square A \text { iff } M, h \Vdash A,
$$

whereas the truth condition for the modal box is

$$
M, w \Vdash \square A \text { iff } M, R(w) \Vdash A .
$$

Despite the differing direction in their accessibility relations, the logics of the two boxes can still coincide.

There is an apparent problem with taking revision sequences to be Kripke models: The initial hypotheses do not have predecessors, so revision sequences, so understood, are not QD! models. This problem is merely apparent. For any given sentence in the language of QD!, only finitely many worlds are used for its evaluation. Similarly, only finitely many stages of a revision sequence are used to evaluate $A$, taking its boxes to be those of revision theory. Since validity can be understood as eventual stable truth, one can always move further down a revision sequence to provide enough predecessor hypotheses to evaluate $A$.

The semantic intuition can be borne out, but it will be helpful, in light of some of the semantic differences indicated above, to consider the connection in terms of the proof theory. Let us turn to a proof system for revision theory.

The system $C_{0}^{\square}$ is a Fitch-style proof system for the extended revision theory. ${ }^{40} C_{0}^{\square}$ uses indexed formulas, $A^{i}$, where $i$ can be any integer. Intuitively, the indices reflect the relative stage at which a sentence is true in a revision sequence, and they restrict when connective rules can be applied.

\footnotetext{
${ }^{40}$ For full details of the proof system for basic revision theory, $C_{0}$, including soundness and completeness, see Gupta and Belnap (1993), although that formulation does not use the rules for $\supset$ and $\vee . C_{0}$ was introduced by Gupta (8 89). $C_{0}^{\square}$ was introduced by Standefer (2015). The proofs of soundness and completeness for $C_{0}^{\square}$ are, in large part, similar to those for $C_{0}$, as indicated by Standefer (2015).
} 
The rules for the classical connectives require that all premises and conclusions have the same index. We have included rules for $=$, which is taken to be in the language of revision theory. The disjunction rules have been omitted.

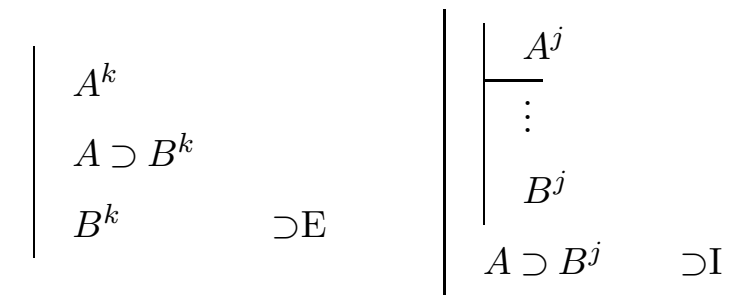

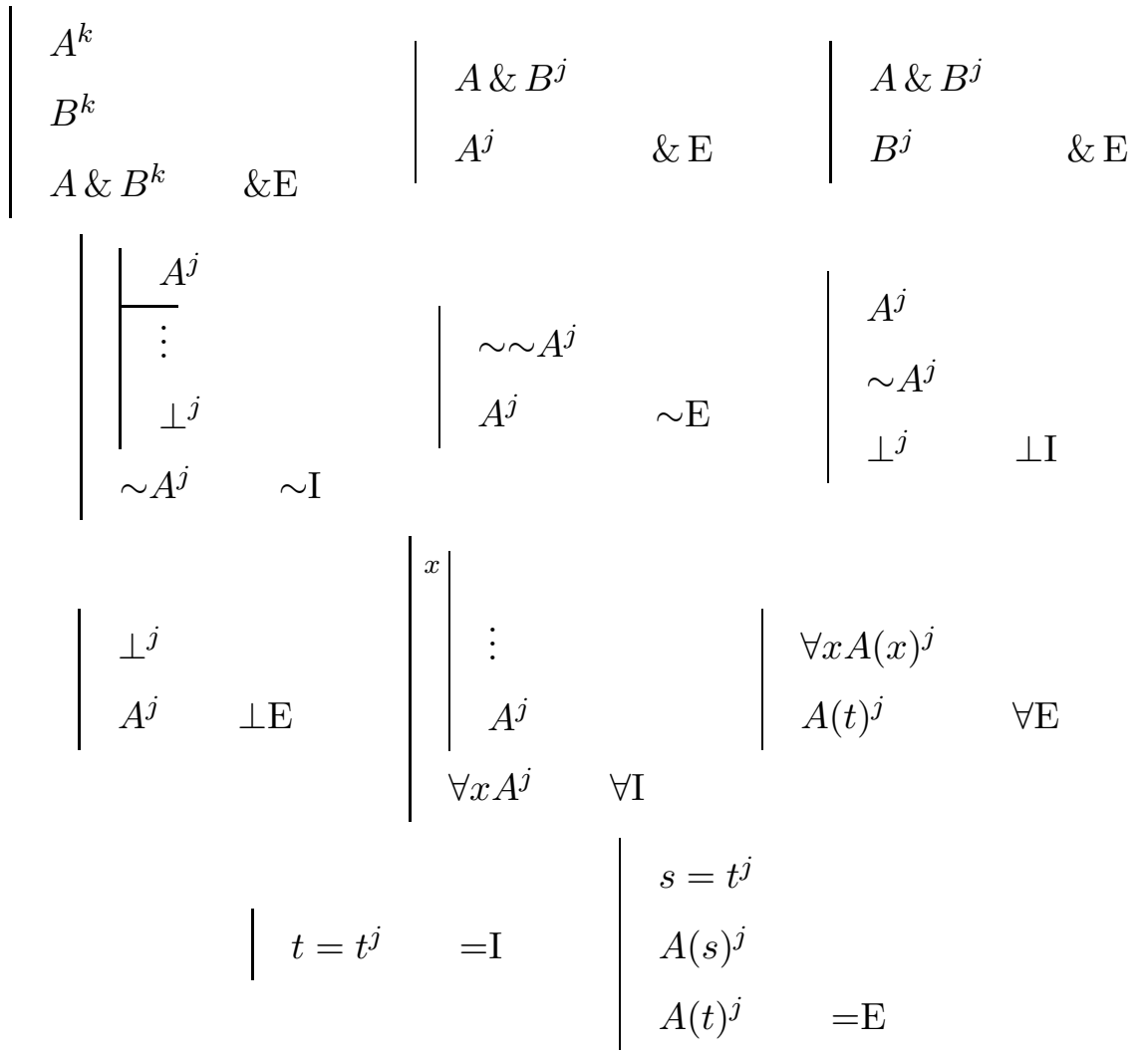

In $\forall \mathrm{E}$ and $=\mathrm{E}, t$ must be free for $x$ and $s$, respectively, in $A$. There are special rules that correspond to the set $\mathscr{D}$ of circular definitions under consideration. The system contains, for each definition, $G x={ }_{D f} A_{G}(x)$, in $\mathscr{D}$, the following pair of rules.

$$
\mid \begin{array}{ll|ll}
A_{G}(t)^{k} & & G t^{k+1} & \\
G t^{k+1} & \text { DefI } & A_{G}(t)^{k} & \text { DefE }
\end{array}
$$


In DefE, $t$ must be free for $x$ in $A_{G}$. The rules for the box also shift the indices.

$$
\begin{array}{ll|ll}
A^{k} & & \square A^{k+1} & \\
\square A^{k+1} & \square \mathrm{I} & A^{k} & \square \mathrm{E}
\end{array}
$$

There is an index shift rule that permits a formula's index to be changed arbitrarily, provided that the formula $B$ contains no defined predicates.

$$
\mid \begin{array}{cc}
B^{j} & \\
B^{k} & \text { IS }
\end{array}
$$

Finally, there is also a rule of assumption and a rule of reiteration that allows one to repeat formulas from open subproofs, with the usual restrictions on reiterating formulas into subproofs flagged with variables. We will use the notation $A_{1}^{j_{1}}, \ldots, A_{n}^{j_{n}} \vdash_{0}^{\mathscr{D}} B^{j_{n+1}}$ to mean that there is a proof of $B^{j_{n+1}}$ from assumptions $A_{1}^{j_{1}}, \ldots, A_{n}^{j_{n}}$ in $C_{0}^{\square}$ with the definition rules for $\mathscr{D}$.

We will make a few brief comments on the semantic intuitions behind the rules of $C_{0}^{\square}$. The definition rules reflect the revision of hypotheses in a revision sequence. If $A_{G}(t)$ holds at one stage, then $G t$ will hold at the next stage. The index shift rule reflects the fact that the interpretation of the base language does not change between revisions. There are restrictions on the indices of the connective and quantifier rules because between stages, the interpretation of some formulas may change. It is not hard to see that permitting the introduction of classical connectives across indices will, in general, be unsound. An interesting caveat arises with respect to $\perp$. It can be introduced with one index, via the $\perp$ I rule, and then, since it contains no defined predicates, its index can be shifted via the IS rule to permit $\sim$ I to be used. With that background in place, let us now turn to the connections.

\subsection{Connections via proofs}

We will now connect $C_{0}^{\square}$ with QD!, or rather QD! theories, in two steps. For the first step, we need a few definitions. First, we add to the language of QD! the propositional constant $\perp$. Let $\mathrm{QD} !_{\perp}$ be QD! with the addition of the usual axioms for $\perp .{ }^{41}$ Next, let $C^{-}$be $C_{0}^{\square}$ without the definition rules, without identity and the identity rules, and with the index shift restricted to just $\perp$. $C^{-}$is $C_{0}^{\square}$ stripped of most of the rules connected to definitions.

\footnotetext{
${ }^{41}$ When we have identity in the language with its own axioms, we take $\perp$ to be $\exists x(x \neq x)$ and omit the subscript.
} 
Index shift for $\perp$ is included so that the negation rules work properly. ${ }^{42}$ The connection between $C^{-}$and $\mathrm{QD} !_{\perp}$ can be established without the additional machinery needed to connect $C_{0}^{\square}$ and the QD! theories. For the first step of the connection, we will show that $C^{-}$and $\mathrm{QD} !_{\perp}$ are equivalent.

For the proofs of the final two theorems, we will need the notion of a $k$-transform.

Definition 1 ( $k$-transform). For $k \geq n$, the $k$-transform of a formula $A^{n}$ is $\square^{k-n} A^{k}$.

Our interest in $k$-transforms comes primarily from the following lemma, whose proof we omit.

Lemma 5. Suppose that there is a deduction $A_{1}^{k_{1}}, \ldots, A_{n}^{k_{n}} \vdash_{0}^{\mathscr{D}} B^{k_{n+1}}$ of an indexed formula from a set of indexed formulas. Let $k=\max \left(\left\{k_{i}: 1 \leq i \leq n+1\right\}\right)$ Let $A_{i}^{\prime}$, respectively $B^{\prime}$, be the $k$-transform of $A_{i}$, respectively $B$. Then the following holds. ${ }^{43}$

$$
A_{1}^{k_{1}}, \ldots, A_{n}^{k_{n}} \vdash_{0}^{\mathscr{D}} B^{k_{n+1}} \text { iff } A_{1}^{\prime}, \ldots, A_{n}^{\prime} \vdash_{0}^{\mathscr{D}} B^{\prime} \text { iff } \vdash_{0}^{\mathscr{D}}\left(\bigwedge_{1 \leq i \leq n} A_{i}^{\prime} \supset B^{\prime}\right)^{k}
$$

If the assumptions of a proof have different indices than the conclusion, the $\supset$ I rule cannot be used. The $k$-transforms, however, can be used to obtain a proof for which one can freely use $\supset$ I to obtain a conditional corresponding, in a sense, to the original proof from assumptions. Note that, while the formulation and proof of the lemma use $C_{0}^{\square}$, the lemma extends to cover $C^{-}$ proofs as well.

Let us say that a $C_{0}^{\square}$, or $C^{-}$, proof is transposed if 0 is the least index appearing in it. The indices in a $C_{0}^{\square}$, or $C^{-}$, proof can be uniformly shifted to obtain a transposed proof. It is clear that, given a proof, there is a unique transposed proof obtained by uniformly shifting indices. We will call this proof the transposition of the original. We can now state and prove our next theorem.

THEOREM 5.1 (Equivalence). The following are equivalent.

1. There is a proof in $C^{-}$of $A^{0}$.

2. There is a proof in $\mathrm{QD} !_{\perp}$ of $A$

\footnotetext{
${ }^{42}$ The use of the index shift rule for $\perp$ can be avoided here by complicating the negation rules slightly.

${ }^{43}$ The rightmost formula exhibits a slight abuse of notation, in that the notation $A^{\prime}$ builds in the index. The indices have been moved to the displayed one.
} 
Proof. The proof of $(2) \Rightarrow(1)$ is straightforward, and we leave it to the reader. We will note that index shift for $\perp$ is sufficient, and seems to be necessary, to obtain $\sim \square^{n} \perp, n \geq 1$.

The proof of $(1) \Rightarrow(2)$ is more involved. Suppose we are given a $C^{-}$proof of $A^{0}$. Let $k$ be the greatest index appearing in its transposition. Replace each line of the transposition with its $k$-transform. The result, while not a proof, can easily be turned into a proof by inserting extra steps adding and removing boxes before applying the rules of the original proof. We then proceed inductively to replace innermost subproofs with the "quasi-proof" method of Anderson and Belnap (1975). We will briefly describe the method. For subproofs not flagged with a variable, the subproof is turned into a sequence of conditionals, the antecedents of which are the assumption of that subproof and the consequents of which are the formulas on the lines of the subproof. Each conditional is then justified by appeal to QD! $\perp$ together with the assumptions of the quasi-proof. For subproofs flagged with a variable $x$, each line is prepended with $\forall x$, inserting parentheses as appropriate, and the result is justified as before. The inductive argument shows how to justify each line of the quasi-proof, permitting the subproof to be eliminated in favor of a sequence of conditionals or quantified formulas. The end result is an $\mathrm{QD} ! \perp$ proof of the desired formula.

We now show how to convert each line of $k$-transformed subproofs into a conditional or quantified formula that can be justified in the manner described. There are many cases, and they are mostly straightforward. We will merely indicate how to fill in a few.

Case: \&E. Suppose that the innermost subproof includes the following steps. Its $k$-transform follows.

$$
\begin{array}{|ll|l}
B^{n} & \square^{k-n} B^{k} \\
\cline { 3 - 3 } & & \vdots \\
C \& D^{m} & & \square^{k-m}(C \& D)^{m} \\
C^{m} & \& \mathrm{E} & \square^{k-m} C^{m} \quad \& \mathrm{E}
\end{array}
$$

We suppose that the steps up to the \&E steps are justified. We then have the following Hilbert-style proof steps.

$$
\mid \begin{array}{ll}
\square^{k-n} B \supset \square^{k-m}(C \& D) & \\
\square^{k-m}(C \& D) \supset \square^{k-m} C & \text { KD! theorem } \\
\square^{k-n} B \supset \square^{k-m} C & \text { transitivity }
\end{array}
$$


Case: $\forall \mathrm{I}$. This case is handled by repeated use of substitution of equivalents and appropriate instances of the Barcan formula. Suppose that the innermost subproof to convert is a categorical proof.

$$
\mid \begin{array}{c|c} 
& \\
\vdots & \\
B^{n} & \\
\forall x B^{n} \quad \forall \mathrm{I}
\end{array}
$$

The innermost subproof is already accounted for by our inductive argument, but it is needed to explain how to handle this case. This subproof is converted to its $k$-transform.

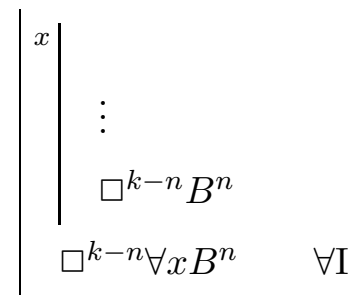

All of the formulas appearing in the innermost subproof are surrounded by parentheses and ' $\forall x$ ' is prepended to them, giving the following.

$$
\mid \begin{aligned}
& \vdots \\
& \forall x\left(\square^{k-n} B\right)^{n} \\
& \square^{k-n} \forall x B^{n}
\end{aligned}
$$

The justification for the last step is $k-n$ uses of the Barcan formula for $B$.

Case: Index shift rule. The justification for this step is that for all $k, m \geq 0, \square^{k} \perp \equiv \square^{m} \perp$ is a theorem of $\mathrm{QD} !_{\perp}$.

This establishes that the $k$-transformation of $A$ is provable, but that sentence may be $\square^{n} A$, for $n \geq 1$. Using completeness, one can show that $\mathrm{QD} !_{\perp}$ is closed under the converse of the rule of Necessitation, namely if $\mathrm{QD} !_{\perp} \vdash \square A$ then $\mathrm{QD} !_{\perp} \vdash A$. Suppose that $\mathrm{QD} !_{\perp} \vdash \square A$ and $\mathrm{QD} !_{\perp} \forall \forall A$. By completeness, there is a model $M$ and world $u$ such that $A$ is false in $w$. Extend $M$ with a world $w$ such that $w R u$. Since $\mathrm{QD} !_{\perp} \vdash \square A, \square A$ is true at $w$, whence $A$ is true at $u$, which is a contradiction. Therefore, $A$ is a theorem of $\mathrm{QD} !_{\perp}$. 
The previous theorem establishes an equivalence between $C^{-}$and $\mathrm{QD} !_{\perp}$, but there is a gap between $C^{-}$and $C_{0}^{\square}$. The latter has rules for identity, rules for definitions, and index shift rules for base language formulas. We can extend $\mathrm{QD} !_{\perp}$ to bring it more fully in line with $C_{0}^{\square}$. First, let $\mathrm{QD} !^{=}$be $\mathrm{QD} !_{\perp}$ with the addition of $=$ to the language and the following axioms. ${ }^{44}$

E1 $t=t$

E2 $s=t \supset\left(A \supset A^{\prime}\right)$, where $A$ and $A^{\prime}$ differ only in that $A^{\prime}$ has $t$ in 0 or more places that $A$ has $s$, assuming $t$ is free for $s$ in $A$

E3 $s \neq t \supset \square(s \neq t)$

Given the other axioms of QD!' ${ }^{=}$E3 implies

$$
\square(s=t) \supset s=t .
$$

Before proceeding, it is worth observing that the analogue of theorem 4.2 does not hold for $\mathrm{QD} !^{=}$. If equality is in the language and treated as logical, then the substitutions will need to replace equality atoms with equality atoms. The problem is that $\mathrm{QD} !^{=}$has as theorems $\forall x \square^{n}(x=x)$, for all $n \geq 0$, but $\forall x \square^{n}(x=x)$ is not a substitution instance of a first-order logical truth if $n \geq 1$. A variation of the theorem that removes boxes on equalities is, however, provable, but we will not go into that further here.

To accommodate the definition and index shift rules in $\mathrm{QD} !^{=}$, we need some additional machinery. With that in place, we can show the relation between an $\mathrm{QD} !^{=}$theory and $C_{0}^{\square}$.

Suppose we have a first-order modal language $\mathscr{L}$ with variables, equality, and possibly infinitely many predicates. Let the set of the predicates in $\mathscr{L}$, including equality, be $\mathcal{A}$. Next, enrich $\mathscr{L}$ with a finite set of unary predicates $\mathcal{B}$, which is disjoint from $\mathcal{A}$. Call the new language $\mathscr{L}^{+}$. For each $G \in \mathcal{B}$, we associate the formula $G(x)$, with the free variable $x$, with a formula of $\mathscr{L}^{+}$, $A_{G}(x)$, that has exactly the variable $x$ free. Let the set of such pairs be $\mathcal{D}$. Let the notation $\bar{x}$ stand for a sequence of variables, $x_{1}, \ldots, x_{n}$, where $n$ is determined by context. We add to QD! ${ }^{=}$, the non-logical axioms $\forall \bar{x}(F \bar{x} \equiv$ $\square F \bar{x})$, for $F \in \mathcal{A}$ and the axioms $\forall x\left(G x \equiv \square A_{G}(x)\right)$, for $\left(G(x), A_{G}(x)\right) \in \mathcal{D}$. We also strengthen the Necessitation rule to apply to theorems whose proofs include the new axioms. ${ }^{45}$ Call the resulting theory $\mathrm{QD} ! \overline{\overline{\mathcal{A}}}^{\mathcal{D}}$.

\footnotetext{
${ }^{44}$ These are taken from Hughes and Cresswell (1996).

${ }^{45}$ This strengthening is along the lines considered by Fitting et al. (1992). For insightful discussion of Necessitation and other rules of proof, see Humberstone (2010).
} 
Next, we need one bit of terminology. Given a set $\mathscr{D}$ of definitions and a set $\mathcal{D}$ of pairs of unary predicate letters and formulas with one free variable, let us say that $\mathscr{D}$, (respectively $\mathcal{D}$ ) agrees with $\mathcal{D}$ (respectively $\mathscr{D}$ ) iff $G x={ }_{D f} A_{G}(x)$ is in $\mathscr{D}$ just in case $\left(G(x), A_{G}(x)\right) \in \mathcal{D}$.

Finally, the result we will prove is the following.

TheOREM 5.2. Let $\mathscr{D}$ be a set of definitions that agrees with $\mathcal{D}$. Then, we have the following.

(1) If $\mathrm{QD} ! \overline{\overline{\mathcal{A}}}_{\mathcal{D}} \vdash A$, then $\vdash_{0}^{\mathscr{D}} A^{0}$.

(2) If $\vdash_{0}^{\mathscr{D}} A^{0}$, then there is a $n \geq 0$ such that $\mathrm{QD} ! \overline{\overline{\mathcal{A}}}, \mathcal{D} \vdash \square^{n} A$.

Proof. For (1), we indicate how $C_{0}^{\square}$ proves the additional axioms added to $\mathrm{QD} !_{\perp}$ to obtain $\mathrm{QD} ! \overline{\overline{\mathcal{A}}}_{\mathcal{D}}$, namely the axioms $\forall \bar{x}(F \bar{x} \equiv \square F \bar{x})$, for $F \in \mathcal{A}$, the biconditionals for the pairs in $\mathcal{D}$, and the identity axioms. The axioms $\forall \bar{x}(F \bar{x} \equiv \square F \bar{x})$ are consequences of the index shift rules. The biconditionals from $\mathcal{D}$ follow immediately from the definition rules together with the box rules. The identity axioms are consequences of the identity and index shift rules. Finally, we note that if $\vdash_{0}^{\mathscr{D}} A^{m}$ for some $m$, then, for all $n, \vdash_{0}^{\mathscr{D}} A^{n}$, since one can uniformly shift indices in the original proof to obtain the desired index on the conclusion.

The strategy for (2) is similar to that of (2) in theorem 5.1. Assume that there is a $C_{0}^{\square}$ proof of $A^{0}$ and that $k$ is the largest index in its transposition. We again use the quasi-proof method of Anderson and Belnap (1975) to show that the transposed $C_{0}^{\square}$ proof can be converted into a Hilbert-style $\mathrm{QD} ! \overline{\bar{A}}_{\mathcal{D}}$ proof. We will indicate how $\mathrm{QD} ! \overline{\overline{\mathcal{A}}}_{\overline{\mathcal{D}}}$ handles the index shift and definition rules. The steps for the rules for identity, the box, and the logical connectives are straightforward.

Case: Index shift. One can show, by induction, that for all formulas $A$ of $\mathscr{L}, \forall \bar{x}(A \equiv \square A)$ is derivable. The base case is immediate from the axioms $\forall \bar{x}(F \bar{x} \equiv \square F \bar{x})$, and the rest by the inductive hypothesis. The theorems $\forall \bar{x}(A \equiv \square A)$ then take care of this case.

Case: DefI. This case is taken care of using the appropriate instances of the axioms $\forall x\left(G x \equiv \square A_{G}(x)\right)$.

Case: DefE. This is similar to the DefI case.

We conclude that, given the assumptions, there is an $n \geq 0$ such that $\mathrm{QD} ! \overline{\overline{\mathcal{A}}}_{\mathcal{D}} \vdash \square^{n} A$.

Theorem 5.2 shows that $\mathrm{QD} ! \overline{\overline{\mathcal{A}}}_{\mathcal{D}}$ can, in a sense, reproduce proofs in $C_{0}^{\square}$. There is a question of whether the theorem can be strengthened so that provability in one system coincides with provability in the other. It 
seems, to us, unlikely that, for all $\mathcal{D}, \mathrm{QD} ! \overline{\bar{A}}_{\mathcal{A}} \mathcal{D}$ is closed under the converse of Necessitation, although there may be special cases in which this works out. It would be useful to pin down for which $\mathcal{D}$ it is the case that $\mathrm{QD} !_{\mathcal{A}, \mathcal{D}}$ is closed under the converse of Necessitation.

\section{Conclusion}

The preceding work shows two things. First, the modal logics KD! and QD! are both pleasantly simple, with theorems 4.1 and 4.2 showing that there is a simple procedure to reduce the modal logic to its classical base. Second, the modal logic QD! represents a kind of modal logical core of extended revision theory, given its tight connection with $C^{-}$. With a caveat, this claim can be extended to the modal theories $\mathrm{QD} ! \overline{\overline{\mathcal{A}}}_{\mathcal{D}} \mathcal{D}$ and $C_{0}^{\square}$, providing another perspective on revision-theoretic validity.

We will close by noting a problem we are leaving open. The formulation of HQD! does not include equality rules. It would be good to be able to add equality to the system in a way that permits an elimination theorem. One possible application of such a system would be serve as the basis of a hypersequent calculus for revision theory, providing a proof system that, unlike $C_{0}^{\square}$, does not use indices. Such a calculus would provide an interesting comparison with the sequent system of Bruni (2013).

Acknowledgements. I am very grateful to Anil Gupta, Nuel Belnap, Greg Restall, Kohei Kishida, and Rohan French for their feedback on various stages of this work. I would also like to thank the anonymous referee for extensive feedback that greatly clarified the paper.

This research was supported by the Australian Research Council, Discovery Grant DP150103801.

\section{References}

Anderson, A. R. and Belnap, N. D. (1975). Entailment: The Logic of Relevance and Necessity, volume 1. Princeton University Press.

Antonelli, G. A. (1994). A revision-theoretic analysis of the arithmetical hierarchy. Notre Dame Journal of Formal Logic, 35(2):204-218.

Avron, A. (1987). A constructive analysis of RM. Journal of Symbolic Logic, 52(4):939-951.

Baillot, P. and Mazza, D. (2010). Linear logic by levels and bounded time complexity. Theoretical Computer Science, 411(2):470 - 503. 
Belnap, N. D. (1962). Tonk, plonk and plink. Analysis, 22(6):130-134.

Bimbó, K. (2014). Proof theory: Sequent calculi and related formalisms. CRC Press.

Blackburn, P., de Rijke, M., and Venema, Y. (2002). Modal Logic. Cambridge Tracts in Theoretical Computer Science. Cambridge University Press.

Boudes, P., Mazza, D., and de Falco, L. T. (2015). An abstract approach to stratification in linear logic. Information and Computation, 241:32 - 61 .

Bruni, R. (2013). Analytic calculi for circular concepts by finite revision. Studia Logica, 101(5):915-932.

Chellas, B. F. (1980). Modal Logic: An Introduction. Cambridge University Press.

Curry, H. B. (1963). Foundations of Mathematical Logic. Dover Publications.

Davies, M. and Humberstone, L. (1980). Two notions of necessity. Philosophical Studies, 38(1):1-31.

Dunn, J. M. (1995). Positive modal logic. Studia Logica, 55(2):301-317.

Dunn, M. and Restall, G. (2002). Relevance logic. In Gabbay, D. and Guenthner, F., editors, Handbook of Philosophical Logic. Kluwer.

Fitting, M. (2014). Nested sequents for intuitionistic logics. Notre Dame Journal of Formal Logic, 55(1):41-61.

Fitting, M. C., Marek, V. W., and Truszczyński, M. (1992). The pure logic of necessitation. Journal of Logic and Computation, 2(3):349-373.

French, R. and Ripley, D. (2015). Contractions of noncontractive consequence relations. Review of Symbolic Logic, 8(3):506-528.

Gabbay, D. M., Kurucz, A., Wolter, F., and Zakharyaschev, M. (2003). Many-Dimensional Modal Logics: Theory and Applications. Elsevier.

Garson, J. W. (2013). Modal Logic for Philosophers. Cambridge University Press, 2nd edition.

Gentzen, G. (1964). Investigations into logical deduction. American Philosophical Quarterly, 1(4):288-306.

Gupta, A. (1988-89). Remarks on definitions and the concept of truth. Proceedings of the Aristotelian Society, 89:227-246. Reprinted in ?, pp. 73-94.

Gupta, A. and Belnap, N. (1993). The Revision Theory of Truth. MIT Press.

Gupta, A. and Standefer, S. (2016). Conditionals in theories of truth. Journal of Philosophical Logic, pages 1-37. Forthcoming. 
Hughes, G. E. and Cresswell, M. J. (1996). A New Introduction to Modal Logic. Routledge.

Humberstone, L. (2010). Smiley's distinction between rules of inference and rules of proof. In Smiley, T. J., Lear, J., and Oliver, A., editors, The Force of Argument: Essays in Honor of Timothy Smiley, pages 107-126. Routledge.

Indrzejczak, A. (2016). Linear time in hypersequent framework. Bulletin of Symbolic Logic, 22(1):121-144.

Kawai, H. (1987). Sequential calculus for a first order infinitary temporal logic. Mathematical Logic Quarterly, 33(5):423-432.

Lahav, O. and Zohar, Y. (2014). SAT-Based Decision Procedure for Analytic Pure Sequent Calculi, pages 76-90. Springer International Publishing, Cham.

Lellmann, B. (2015). Linear nested sequents, 2-sequents and hypersequents. In De Nivelle, H., editor, Automated Reasoning with Analytic Tableaux and Related Methods: 24th International Conference, TABLEAUX 2015, Wroclaw, Poland, September 21-24, 2015, Proceedings, pages 135-150. Springer International Publishing.

Masini, A. (1992). 2-sequent calculus: A proof theory of modalities. Annals of Pure and Applied Logic, 58(3):229-246.

Merz, S. (1992). Decidability and incompleteness results for first-order temporal logics of linear time. Journal of Applied Non-Classical Logics, 2(2):139-156.

Minc, G. (1971). On Some Calculi of Modal Logic. In Orevkov, V., editor, The Calculi of Symbolic Logic. I., volume 98, pages 97-124. American Mathematical Society. Originally published in Russian in 1968 in Proceedings of the Steklov Institute of Mathematics, edited by I. G. Petrovskii and S. M. Nikol'skii.

Negri, S. and von Plato, J. (2001). Structural Proof Theory. Cambridge.

Parsons, J. (2013). Command and consequence. Philosophical Studies, 164(1):61-92.

Poggiolesi, F. (2010). Gentzen Calculi for Modal Propositional Logic. Springer.

Pottinger, G. (1983). Uniform cut-free formulations of T, S4 and S5 (abstract). Journal of Symbolic Logic, 48(3):900-901.

Rescher, N. and Urquhart, A. (1971). Temporal Logic. Springer Verlag. 
Restall, G. (1999). Negation in relevant logics (how I stopped worrying and learned to love the Routley star). In Gabbay, D. M. and Wansing, H., editors, What is Negation?, pages 53-76. Kluwer Academic Publishers.

Restall, G. (2000). An Introduction to Substructural Logics. Routledge.

Restall, G. (2012). A cut-free sequent system for two-dimensional modal logic, and why it matters. Annals of Pure and Applied Logic, 163(11):16111623.

Segerberg, K. (1986). Modal logics with functional alternative relations. Notre Dame Journal of Formal Logic, 27(4):504-522.

Standefer, S. (2015). Solovay-type theorems for circular definitions. Review of Symbolic Logic, 8(3):467-487.

Troelstra, A. S. and Schwichtenberg, H. (2000). Basic Proof Theory. Cambridge University Press.

Wansing, H. (1998). Displaying Modal Logic. Kluwer.

ShaWn Standefer

School of Historical and Philosophical Studies

The University of Melbourne

Parkville 3010, Australia

sstandefer@unimelb.edu.au 